\title{
Three-Boson Spectrum in the Presence of 1D Spin-Orbit Coupling: Efimov's Generalized Radial Scaling Law
}

\author{
Q. Guan and D. Blume \\ Homer L. Dodge Department of Physics and Astronomy, The University of Oklahoma, \\ 440 West Brooks Street, Norman, Oklahoma 73019, USA
}

(Received 26 October 2017; revised manuscript received 6 March 2018; published 1 June 2018)

\begin{abstract}
Spin-orbit-coupled cold-atom systems, governed by Hamiltonians that contain quadratic kinetic energy terms typical for a particle's motion in the usual Schrödinger equation and linear kinetic energy terms typical for a particle's motion in the usual Dirac equation, have attracted a great deal of attention recently since they provide an alternative route for realizing fractional quantum Hall physics, topological insulators, and spintronics physics. The present work focuses on the three-boson system in the presence of 1D spinorbit coupling, which is most relevant to ongoing cold-atom experiments. In the absence of spin-orbitcoupling terms, the three-boson system exhibits the Efimov effect: the entire energy spectrum is uniquely determined by the $s$-wave scattering length and a single three-body parameter; i.e., using one of the energy levels as input, the other energy levels can be obtained via Efimov's radial scaling law, which is intimately tied to a discrete scaling symmetry. It is demonstrated that the discrete scaling symmetry persists in the presence of 1D spin-orbit coupling, implying the validity of a generalized radial scaling law in fivedimensional space. The dependence of the energy levels on the scattering length, spin-orbit-coupling parameters, and center-of-mass momentum is discussed. It is conjectured that three-body systems with other types of spin-orbit-coupling terms are also governed by generalized radial scaling laws, provided the system exhibits the Efimov effect in the absence of spin-orbit coupling.
\end{abstract}

\section{INTRODUCTION}

Under which conditions do two, three, or more particles form weakly bound states, i.e., bound states that are larger than the range of the two-, three-, and higher-body forces that bind the particles together? And under which conditions are the characteristics of these few-body bound states governed by underlying symmetries? These questions are of utmost importance across physics. For example, the existence of bound tetraquark systems [1], first proposed in 1964 by Gell-Mann [2], has been challenging our understanding of QCD. The existence of the extremely weakly bound triton has a profound effect on the nuclear chart, including the existence of larger exotic halo nuclei $[3,4]$. Historically, the triton has played an important role in the context of the Thomas collapse [5] and the Efimov effect $[6,7]$, which is intimately tied to a discrete scaling symmetry of the three-body Schrödinger equation.

The three-boson system with two-body short-range interactions is considered the holy grail of few-body

Published by the American Physical Society under the terms of the Creative Commons Attribution 4.0 International license. Further distribution of this work must maintain attribution to the author(s) and the published article's title, journal citation, and DOI. physics. It has captured physicists' attention since Efimov's bizarre and counterintuitive predictions in the early 1970s [6,7] and has spurred a flurry of theoretical and experimental works from nuclear to atomic to condensed matter to particle physics [8-21]. The unique scaling laws exhibited by Efimov trimers can be traced back to the existence of just one large length scale in the problem, namely the two-body $s$-wave scattering length. The main focus of the present work is on investigating what happens to the three-boson Efimov states in the presence of 1D spinorbit coupling. Similar to few-body systems on the lattice [22], the 1D spin-orbit coupling introduces a parametric dependence of the relative Hamiltonian on the center-ofmass momentum. This center-of-mass momentum dependence leads, as we show, to a modification of the lowest break-up threshold and has a profound effect on the binding energy. Despite this dependence on the center-of-mass momentum and despite the fact that the spin-orbit-coupling terms depend on three additional parameters (namely, $k_{\mathrm{so}}$, $\Omega$ and $\delta$; see below), it is argued that the three-boson system in the presence of 1D spin-orbit coupling possesses, in the zero-range limit, a discrete scaling symmetry and it is shown that the energy spectrum is described by a generalized radial scaling law.

The 1D spin-orbit-coupling terms, which break the rotational symmetry, introduce an unusual single-particle 
dispersion. The Hamiltonian $\hat{H}_{j}$ of the $j$ th particle with mass $m$ and momentum operator $\hat{\vec{p}}_{j}$ (with components $\hat{p}_{j, x}, \hat{p}_{j, y}$, and $\left.\hat{p}_{j, z}\right)$ is not simply given by $\hat{\vec{p}}_{j}^{2} /(2 m)$ but includes a term that emulates a spin- $1 / 2$ particle interacting with a momentum-dependent "magnetic field" of infinite range [23-27]:

$$
\hat{H}_{j}=\frac{\hat{\vec{p}}_{j}^{2}}{2 m} I_{j}+\hat{\vec{B}}\left(\hat{p}_{j, z}\right) \cdot \hat{\vec{\sigma}}_{j} .
$$

Here, $I_{j}$ denotes the $2 \times 2$ identity matrix that spans the spin degrees of freedom of the $j$ th particle, the vector $\hat{\vec{\sigma}}_{j}$ contains the three Pauli matrices $\hat{\sigma}_{j, x}, \hat{\sigma}_{j, y}$, and $\hat{\sigma}_{j, z}$ of the $j$ th particle, and $\hat{\vec{B}}$ represents the effective magnetic field, $\hat{\vec{B}}=\left(\Omega / 2,0, \hbar k_{\mathrm{so}} \hat{p}_{j, z} / m+\delta / 2\right)$, felt by the $j$ th particle. The Raman coupling $\Omega$, detuning $\delta$, and spin-orbitcoupling strength $k_{\text {so }}$, which characterize the two-photon Raman transition that couples (effectively) two hyperfine states of an ultracold atom, describe the deviations from the "normal" quadratic single-particle dispersion curves,

$$
E_{j, \pm}=\frac{\vec{p}_{j}^{2}}{2 m} \pm \sqrt{\left(\frac{\hbar k_{\mathrm{so}} p_{j, z}}{m}+\frac{\delta}{2}\right)^{2}+\frac{\Omega^{2}}{4}}
$$

where $\vec{p}_{j}$ and $p_{j, z}$ (both without "hat") are expectation values of the corresponding operators. For large $\left|\vec{p}_{j}\right|$, the dispersion curves $E_{j, \pm}$ approach $\vec{p}_{j}^{2} /(2 m)$. For small $\left|\vec{p}_{j}\right|$, in contrast, the $E_{j, \pm}$ curves deviate appreciably from $\vec{p}_{j}^{2} /(2 m)$. The momenta $\vec{p}_{j}$ are generalized momenta (sometimes also referred to as quasimomenta) and not mechanical momenta (sometimes also referred to as kinetic momenta) [28]. Throughout this article, we frequently drop the prefix "generalized" and refer to $\vec{p}_{j}$ as momentum vector of the $j$ th atom. The Hamiltonian given in Eq. (1) can also be realized by lattice shaking techniques as well as in photonic crystals and mechanical setups [27,29-31].

If two-body short-range interactions are added, the modified single-particle dispersion curves can significantly alter the properties of weakly bound two- and three-body states. This has been demonstrated extensively for two identical fermions for 1D, 2D, and 3D spin-orbit coupling [32-41] and for two identical bosons for 2D and 3D spinorbit coupling [41-45] but not for the 1D spin-orbit coupling considered in this work. The present work presents the first study of how the experimentally most frequently realized 1D spin-orbit-coupling terms modify the three-boson energy spectrum. We note, however, that several three-body studies for bosonic and fermionic systems with other types of spin-orbit coupling exist [46-49]. All of these earlier studies limited themselves to vanishing center-of-mass momentum. Our work, in contrast, allows for finite center-of-mass momenta.
The key objective of the present work is to show that the three-boson system in the presence of 1D spin-orbit coupling obeys a generalized radial scaling law, which reflects the existence of a discrete scaling symmetry in the limit of zero-range interactions. The scaling parameter $\lambda_{0}$, $\lambda_{0} \approx 22.694$, is the same as in the absence of the spin-orbitcoupling terms. The generalized radial scaling law relates the energy for a given $1 / a_{s}, k_{\mathrm{so}}, \Omega$, and $\tilde{\delta}[\tilde{\delta}$ is a generalized detuning that is defined in terms of the detuning $\delta$ and the $z$ component of the center-of-mass momentum; see Eq. (21)] to the energy for a scaled set of parameters, namely $\lambda_{0} / a_{s}$, $\lambda_{0} k_{\mathrm{so}},\left(\lambda_{0}\right)^{2} \Omega$, and $\left(\lambda_{0}\right)^{2} \tilde{\delta}$. Correspondingly, the term "radial" does not refer to the radius in a two-dimensional space as in the usual Efimov scenario but to the radius in a five-dimensional space. The fact that the discrete scaling symmetry "survives" when the spin-orbit-coupling terms are added to the three-boson Hamiltonian with zero-range interactions can be intuitively understood from the observation that $k_{\mathrm{so}}, \Omega$, and $\tilde{\delta}$ can be thought of as introducing finite length scales into the system. In the standard Efimov scenario, $a_{s}$ introduces a finite length scale and the radial scaling law holds regardless of whether $\left|a_{s}\right|$ is larger or smaller than the size of the trimer, provided $\left|a_{s}\right|$ is much larger than the intrinsic scales of the underlying two- and three-body interactions. In the generalized Efimov scenario considered here, the parameters $a_{s}, k_{\mathrm{so}}, \Omega$, and $\tilde{\delta}$ each introduce a finite length scale. Correspondingly, the generalized radial scaling law holds regardless of whether these length scales are larger or smaller than the size of the trimer, provided the length scales are much larger than the intrinsic scales of the underlying two- and three-body interactions.

Our findings for the experimentally most frequently realized 1D spin-orbit coupling are consistent with Ref. [48]. References [46,48] considered an impurity with 3D spin-orbit coupling that interacts with two identical fermions that do not feel any spin-orbit-coupling terms and interact with the impurity through short-range two-body potentials. Restricting themselves to vanishing center-ofmass momenta, Ref. [46] stated that the trimers for mass ratio $\gtrsim 13.6$ "no longer obey the discrete scaling symmetry even at resonance" because the spin-orbit coupling "introduces an additional length scale." In Ref. [48], the same authors arrive at a seemingly different conclusion, namely, "in the presence of SO [spin-orbit] coupling, the system exhibits a discrete scaling behavior" and "the scaling ratio is identical to that without SO [spin-orbit] coupling." The two statements can be reconciled by noting that the discrete scaling symmetry requires an enlarged parameter space, an aspect that was recognized in Ref. [48] but not in Ref. [46]. We conjecture that the discrete scaling symmetry holds for any type of spin-obit coupling and all center-of-mass momenta. Depending on the type of the spin-orbit coupling, the generalized Efimov plot is four or five dimensional and the generalized radial scaling law applies to the entire low-energy spectrum. The dependence of the energy 
levels on the system parameters has to be calculated explicitly once for each type of spin-obit coupling.

The remainder of this article is organized as follows. To set the stage, Sec. II reviews the standard Efimov scenario for three identical bosons. Section III introduces the system Hamiltonian in the presence of 1D spin-orbit coupling and discusses the associated continuous and discrete scaling symmetries. The generalized radial scaling law for the three-boson system in the presence of 1D spin-orbit coupling is confirmed numerically in Sec. IV. Section V highlights the role of the center-of-mass momentum and discusses possible experimental signatures of this dependence. Finally, Sec. VI presents an outlook. Technical details are relegated to several appendixes.

\section{REVIEW OF STANDARD EFIMOV SCENARIO}

The relative Hamiltonian for two identical bosons of mass $m$ interacting through the zero-range contact interaction $V_{2 \mathrm{~b}, \mathrm{zr}}(\vec{r})$,

$$
V_{2 \mathrm{~b}, \mathrm{zr}}(\vec{r})=\frac{4 \pi \hbar^{2} a_{s}}{m} \delta^{(3)}(\vec{r}) \frac{\partial}{\partial r} r,
$$

where $a_{s}$ denotes the two-body $s$-wave scattering length and $\vec{r}$ the internuclear distance vector $(r=|\vec{r}|)$, possesses a continuous scaling symmetry [8]. Performing the transformation

$$
a_{s} \rightarrow \lambda a_{s}, \quad \vec{r} \rightarrow \lambda \vec{r} \quad \text { and } \quad t \rightarrow \lambda^{2} t,
$$

where $t$ denotes the time and $\lambda$ a real number (scaling parameter), the relative two-body time-dependent Schrödinger equation remains unchanged.

Importantly, the continuous scaling symmetry extends to three identical mass $m$ bosons with position vectors $\vec{r}_{j}$ that interact through pairwise $s$-wave zero-range interactions $V_{2 \mathrm{~b}, \mathrm{zr}}\left(\vec{r}_{j k}\right)$ [8]. To see this, we consider the timedependent Schrödinger equation for the relative three-body Hamiltonian $\hat{H}_{\text {rel }}$,

$\hat{H}_{\text {rel }}=\sum_{j=1,2}-\frac{\hbar^{2}}{2 \mu_{j}} \nabla_{\vec{\rho}_{j}}^{2}+\sum_{j=1}^{2} \sum_{k=j+1}^{3} V_{2 \mathrm{~b}, \mathrm{zr}}\left(\vec{r}_{j k}\right)+V_{3 \mathrm{~b}, \mathrm{zr}}(R)$,

where $\vec{\rho}_{j}$ denotes the $j$ th relative Jacobi vector and $\mu_{j}$ the associated Jacobi mass. We use a " $K$ tree" (see Appendix A) in which $\mu_{1}$ for the two-body system is given by $m / 2$ and $\mu_{1}$ and $\mu_{2}$ for the three-body system are given by $m / 2$ and $2 m / 3$. The zero-range three-body potential $V_{3 \mathrm{~b}, \mathrm{zr}}(R)$,

$$
V_{3 \mathrm{~b}, \mathrm{zr}}(R)=g_{3} \frac{\hbar^{2}}{m} \delta^{(6)}(R)
$$

is written in terms of a six-dimensional delta function in the three-body hyperradius $R, R^{2}=r_{12}^{2}+r_{13}^{2}+r_{23}^{2}$. Since the coupling constant $g_{3}$ has units of length ${ }^{4}$, it can be rewritten as $g_{3}=C \kappa_{*}^{-4}$, where $C$ is a real constant and $\kappa_{*}$ the threebody binding momentum of one of the three-boson bound states at unitarity (infinite $a_{s}$ ). While $V_{3 \mathrm{~b}, z \mathrm{r}}(R)$ has to be regularized in practice, the explicit regularization is irrelevant for our purpose. Performing the transformation

$a_{s} \rightarrow \lambda a_{s}, \quad \vec{r}_{j k} \rightarrow \lambda \vec{r}_{j k}, \quad t \rightarrow \lambda^{2} t, \quad$ and $\kappa_{*} \rightarrow \lambda^{-1} \kappa_{*}$,

the Schrödinger equation for the relative Hamiltonian given in Eq. (5) remains unchanged; i.e., the three-body system possesses a continuous scaling symmetry.

Intriguingly, the three-body system with zero-range interactions additionally exhibits an exact discrete scaling symmetry [8]. The discrete transformation is given by

$a_{s} \rightarrow\left(\lambda_{0}\right)^{n} a_{s}, \quad \vec{r}_{j k} \rightarrow\left(\lambda_{0}\right)^{n} \vec{r}_{j k}, \quad t \rightarrow\left(\lambda_{0}\right)^{2 n} t, \quad$ and $\kappa_{*} \rightarrow \kappa_{*}$,

where $n= \pm 1, \pm 2, \ldots, \pm \infty$ and $\lambda_{0} \approx 22.694$. The discrete scaling transformation, which underlies the three-body Efimov effect, is illustrated in Fig. 1(a). Fixing the three-body parameter $\kappa_{*}$ [see Eq. (8)], the Efimov plot depicts $K$ as a function of $1 / a_{s}$, where

$$
K=-\sqrt{m|E| / \hbar^{2}},
$$

and $E$ denotes the eigenenergy of the Hamiltonian $\hat{H}_{\text {rel }}$ given in Eq. (5). The thick solid line in Fig. 1(a) shows $K$ for one of the three-body eigenenergies. The thick solid line merges with the three-atom threshold on the negative $a_{s}$ side and with the atom-dimer threshold (dashed line) on the positive $a_{s}$ side. The thick solid line is obtained by solving the time-independent Schrödinger equation for the threebody Hamiltonian $\hat{H}_{\text {rel }}$. Provided the thick solid line is known (a parametrization can be found in Refs. $[8,11]$ ), the thin solid lines-which correspond to other threebody eigenenergies - can be obtained using the discrete scaling symmetry without having to explicitly solve the Schrödinger equation again. For the construction, it is convenient to switch from the vector $\vec{y}=\left(1 / a_{s}, K\right)^{T}$ to a radius $y=|\vec{y}|$ and an angle $\xi$,

$$
K=-y \sin \xi
$$

and

$$
\left(a_{s}\right)^{-1}=y \cos \xi
$$

where $\xi$ goes from $\pi / 4$ to $\pi$. The limits $\pi / 4$ and $\pi$ are set by the atom-dimer and three-atom thresholds, respectively. To obtain the thin solid lines in Fig. 1(a) from the thick solid 


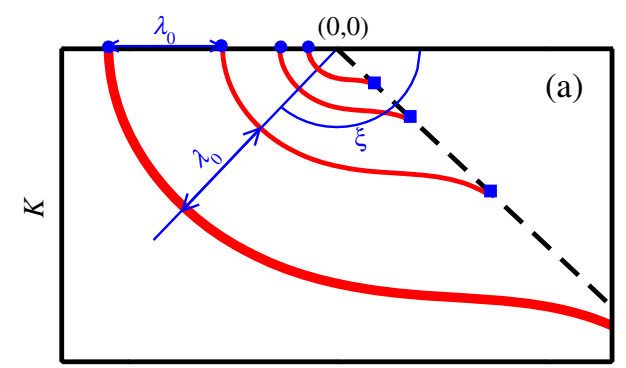

$1 / a_{s}$

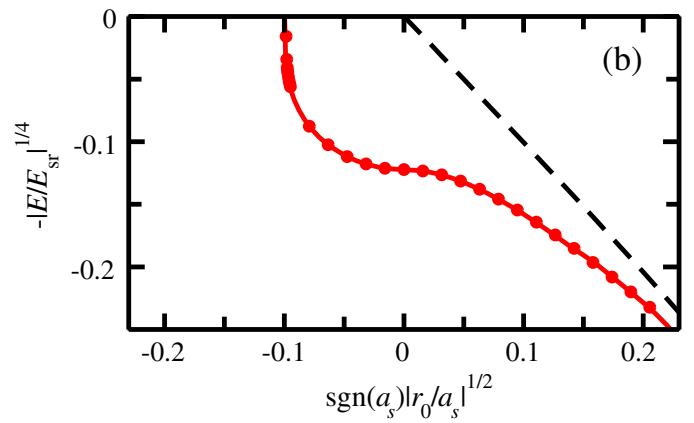

FIG. 1. Radial scaling law for the standard Efimov scenario. (a) The solid lines show the quantity $K$ as a function of $1 / a_{s}$ for the zero-range three-boson Hamiltonian. To make this plot, $\lambda_{0}$ has been artificially set to 2 instead of 22.694. The dashed line shows the atom-dimer threshold. The thin radially outgoing solid lines and arrows illustrate the scaling law. Circles and squares mark the critical scattering lengths $a_{-}$at which the trimer energy is degenerate with the three-atom threshold and the critical scattering lengths $a_{*}$ at which the trimer energy is degenerate with the atom-dimer threshold, respectively. (b) Collapse of neighboring energy levels for the finite-range interaction model $\left[\hat{H}_{\text {rel }}\right.$ in Eq. (5) with $V_{2 \mathrm{~b}, \mathrm{zr}}$ and $V_{3 \mathrm{~b}, \mathrm{zr}}$ replaced by $V_{2 \mathrm{~b}, \mathrm{G}}$ and $V_{3 \mathrm{~b}, \mathrm{G}}$, respectively; $R_{0}=\sqrt{8} r_{0}$ and $\left.\left(\kappa_{*}\right)^{-1} \approx 66.05 r_{0}\right]$. The solid line shows the fourth root of the energy of the lowest three-boson state as a function of the square root of the inverse of the $s$-wave scattering length. The dashed line shows the associated atomdimer threshold. The dots show the energy of the second-lowest three-boson state, with the radial scaling law applied in reverse so as to collapse the second-lowest level (dots) onto the lowest level (solid line). For clarity, the scaled atom-dimer threshold for the second-lowest three-boson state is not shown.

line, one fixes the angle $\xi$ and reads off the values of the pair $\left(1 / a_{s}, K\right)$ corresponding to the solid line. Using

$$
y=\sqrt{\left(a_{s}\right)^{-2}+K^{2}},
$$

it can be seen that the discrete scaling transformation $a_{s} \rightarrow\left(\lambda_{0}\right)^{n} a_{s}$ and $E \rightarrow\left(\lambda_{0}\right)^{-2 n} E$ implies $y \rightarrow\left(\lambda_{0}\right)^{-n} y$. Thus, dividing the radius $y$ of the thick solid line by $\left(\lambda_{0}\right)^{ \pm 1},\left(\lambda_{0}\right)^{ \pm 2}, \ldots$ and using the scaled value of $y$ in Eqs. (10) and (11), one obtains the values of the vectors $\vec{y}=\left(1 / a_{s}, K\right)^{T}$ corresponding to the thin solid lines. This construction, referred to as Efimov's radial scaling law, is a direct consequence of the discrete scaling symmetry. If the three-boson system is characterized by $\kappa_{*}^{\text {new }}$ instead of $\kappa_{*}$, the entire energy spectrum is scaled; i.e., if $\vec{y}=\left(1 / a_{s}, K\right)^{T}$ describes a point on the Efimov plot for $\kappa_{*}$, then $\left(\kappa_{*}^{\text {new }} / \kappa_{*}\right) \vec{y}$ describes a point on the Efimov plot for $\kappa_{*}^{\text {new }}$.

\section{SYMMETRIES IN THE PRESENCE OF 1D SPIN-ORBIT COUPLING}

This section generalizes the symmetry discussion presented in the previous section to the two- and three-boson systems in the presence of 1D spin-obit coupling. As a first step, we derive the relative two- and three-body Hamiltonian with zero-range interactions in the presence of 1D spin-orbit coupling. In a second step, we show that these systems possess a continuous scaling symmetry. In a third step, we argue that the three-boson system additionally exhibits a discrete scaling symmetry, suggesting the existence of a generalized radial scaling law. Numerical evidence that supports our claim that the three-boson system with 1D spin-orbit coupling is governed by a generalized radial scaling law is presented in Sec. IV.

We start with the first step. The $N$-boson Hamiltonian in the presence of $1 \mathrm{D}$ spin-orbit coupling reads

$$
\hat{H}=\hat{H}_{\mathrm{ni}}+\hat{V}_{\mathrm{int}},
$$

where the noninteracting and interacting pieces are given by

$$
\begin{aligned}
\hat{H}_{\mathrm{ni}}= & \sum_{j=1}^{N} \frac{\hat{\vec{p}}_{j}^{2}}{2 m} I_{1, \ldots, N} \\
& +\sum_{j=1}^{N}\left(\frac{\hbar k_{\mathrm{so}}}{m} \hat{p}_{j, z}+\frac{\delta}{2}\right) I_{1, \ldots, j-1} \hat{\sigma}_{j, z} I_{j+1, \ldots, N} \\
& +\sum_{j=1}^{N} \frac{\Omega}{2} I_{1, \ldots, j-1} \hat{\sigma}_{j, x} I_{j+1, \ldots, N}
\end{aligned}
$$

and

$\hat{V}_{\text {int }}=\left(\sum_{j=1, j<k}^{N} V_{2 \mathrm{~b}}\left(r_{j k}\right)+\sum_{j=1, j<k<l}^{N} V_{3 \mathrm{~b}}\left(r_{j k l}\right)\right) I_{1, \ldots, N}$.

Here, $I_{j, \ldots, k}$ with $j<k$ spans the spin degrees of freedom of particles $j$ through $k, I_{j, \ldots, k}=I_{j} \otimes \ldots \otimes I_{k}$. For $N=2$, only $V_{2 \mathrm{~b}}$ contributes. For $N=3, r_{j k l}$ is equal to the threebody hyperradius $R$. The interaction model considered throughout this work assumes that the interactions are the same in all spin channels.

It is, just as in the case without spin-orbit coupling, convenient to use Jacobi coordinates $\vec{\rho}_{j}$ and associated momentum operators $\hat{\vec{q}}_{j}$ instead of the single-particle quantities $\vec{r}_{j}$ and $\hat{\vec{p}}_{j}$. Importantly, the $N$ th Jacobi 
"quantities" $\vec{\rho}_{N}$ and $\hat{\vec{q}}_{N}$ correspond to the center-of-mass vector and center-of-mass momentum operator. It can be shown straightforwardly that the Hamiltonian $\hat{H}$ commutes with the center-of-mass momentum operator $\hat{\vec{q}}_{N}$ [50]; i.e., the Schrödinger equation $\hat{H} \Psi=E \Psi$ can be solved for each fixed $\vec{q}_{N}$. Using this and Jacobi coordinates, the noninteracting fixed- $\vec{q}_{N}$ Hamiltonian, denoted by $\hat{\bar{H}}_{\text {ni }}$, reads

$$
\hat{\bar{H}}_{\mathrm{ni}}=\hat{\bar{H}}_{\mathrm{ni}, \mathrm{rel}}+\frac{\vec{q}_{N}^{2}}{2 \mu_{N}} I_{1, \ldots, N},
$$

where

$$
\begin{aligned}
\hat{\bar{H}}_{\mathrm{ni}, \mathrm{rel}}= & \sum_{j=1}^{N-1} \frac{\hat{\vec{q}}_{j}^{2}}{2 \mu_{j}} I_{1, \ldots, N}+\sum_{j=1}^{N-1} \frac{\hbar k_{\mathrm{so}}}{m} \hat{q}_{j, z} \hat{\Sigma}_{j, z} \\
& +\sum_{j=1}^{N} \frac{\Omega}{2} I_{1, \ldots, j-1} \hat{\sigma}_{j, x} I_{j+1, \ldots, N}+\left(\frac{\hbar k_{\mathrm{so}}}{\mu_{N}} q_{N, z}+\frac{\delta}{2}\right) \\
& \times\left(\sum_{j=1}^{N} I_{1, \ldots, j-1} \otimes \hat{\sigma}_{j, z} \otimes I_{j+1, \ldots, N}\right) .
\end{aligned}
$$

The explicit form of the operators $\hat{\Sigma}_{j, z}$ with $j=1, \ldots, N-1$ is given in Appendix A. Note that the first and second terms on the right hand side of Eq. (17) contain momentum operators while the fourth term on the right hand side of Eq. (17) and the second term on the right-hand side of Eq. (16) contain expectation values of the center-of-mass momentum operators (and not operators). As "usual," the interaction $\hat{V}_{\text {int }}$ depends on $\vec{\rho}_{1}, \ldots, \vec{\rho}_{N-1}$ but not on the center-of-mass vector $\vec{\rho}_{N}$. This implies that the eigenstates $\Psi$ can be written as

$$
\Psi=\Phi_{\text {c.m. }} \Phi_{\text {rel }},
$$

where [51]

$$
\Phi_{\mathrm{c.m.}}=\exp \left(\frac{l \vec{q}_{N} \cdot \vec{\rho}_{N}}{\hbar}\right)
$$

and where the $\Phi_{\text {rel }}$, which are eigenstates of $\hat{\bar{H}}_{\text {rel }}$,

$$
\hat{\bar{H}}_{\text {rel }}=\hat{\bar{H}}_{\text {ni,rel }}+\hat{V}_{\text {int }},
$$

depend on the Jacobi vectors $\vec{\rho}_{1}, \ldots, \vec{\rho}_{N-1}$ and the spin degrees of freedom.

Equation (17) shows that the eigenenergies of $\hat{H}$ depend on the generalized detuning $\tilde{\delta}$,

$$
\frac{\tilde{\delta}}{2}=\frac{\hbar k_{\mathrm{so}}}{\mu_{N}} q_{N, z}+\frac{\delta}{2}
$$

i.e., $q_{N, z}$ and $\delta$ enter as a combination and not as independent parameters. This observation suggests that the center-of-mass momentum may play a decisive role in determining the characteristics of the weakly bound two- and three-body states (see also Refs. [35,37]). The parametric dependence of the Hamiltonian $\hat{\bar{H}}_{\text {rel }}$ on the $z$ component $q_{N, z}$ of the center-of-mass momentum is a direct consequence of the fact that the presence of the spin-orbit coupling breaks the Galilean invariance [26]. One immediate consequence of the broken Galilean invariance is that knowing the energy of an eigenstate with $q_{N, z}=0$ does not, in general, suffice for predicting the energy of an eigenstate with $q_{N, z} \neq 0$. Importantly, the eigenstates $\Psi$ depend, in general, explicitly on $q_{N, z}$ and $\delta$ and not just on $\tilde{\delta}$.

We are now ready to address the second step. Parametrizing the two-body interactions $V_{2 \mathrm{~b}}$ by the zerorange potential $V_{2 \mathrm{~b}, \mathrm{zr}}$, the $N=2$ relative Hamiltonian depends on four parameters, namely, $a_{s}, k_{\mathrm{so}}, \Omega$, and $\tilde{\delta}$. It can be readily checked that the corresponding timedependent Schrödinger equation is invariant under the transformation

$$
\begin{array}{cc}
a_{s} \rightarrow \lambda a_{s}, & k_{\mathrm{so}} \rightarrow \lambda^{-1} k_{\mathrm{so}}, \quad \Omega \rightarrow \lambda^{-2} \Omega, \\
\tilde{\delta} \rightarrow \lambda^{-2} \tilde{\delta}, & \vec{r} \rightarrow \lambda \vec{r}, \quad \text { and } t \rightarrow \lambda^{2} t ;
\end{array}
$$

i.e., the $N=2$ system with zero-range interactions possesses a continuous scaling symmetry. The continuous scaling symmetry extends to the three-boson system with zero-range interactions $\left[V_{2 \mathrm{~b}}=V_{2 \mathrm{~b}, \mathrm{zr}}\right.$ and $V_{3 \mathrm{~b}}=V_{3 \mathrm{~b}, \mathrm{zr}}$ in Eq. (15)] in the presence of spin-orbit coupling since the corresponding time-dependent $N=3$ Schrödinger equation is invariant under the transformation

$$
\begin{aligned}
& a_{s} \rightarrow \lambda a_{s}, \quad k_{\mathrm{so}} \rightarrow \lambda^{-1} k_{\mathrm{so}}, \quad \Omega \rightarrow \lambda^{-2} \Omega, \quad \tilde{\delta} \rightarrow \lambda^{-2} \tilde{\delta}, \\
& \vec{r} \rightarrow \lambda \vec{r}, \quad t \rightarrow \lambda^{2} t, \quad \text { and } \kappa_{*} \rightarrow \lambda^{-1} \kappa_{*} .
\end{aligned}
$$

Equations (22) and (23) generalize Eqs. (4) and (7) from Sec. II.

Paralleling the discussion of Sec. II, step 3 poses the question of whether or not the three-boson system in the presence of spin-orbit coupling additionally possesses a discrete scaling symmetry in the zero-range interaction limit. Our claim is that it does and that the discrete transformation is given by

$$
\begin{aligned}
a_{s} & \rightarrow\left(\lambda_{0}\right)^{n} a_{s}, \quad k_{\mathrm{so}} \rightarrow\left(\lambda_{0}\right)^{-n} k_{\mathrm{so}}, \quad \Omega \rightarrow\left(\lambda_{0}\right)^{-2 n} \Omega, \\
\tilde{\delta} & \rightarrow\left(\lambda_{0}\right)^{-2 n} \tilde{\delta}, \quad \vec{r} \rightarrow\left(\lambda_{0}\right)^{n} \vec{r}, \quad t \rightarrow\left(\lambda_{0}\right)^{2 n} t, \quad \text { and } \kappa_{*} \rightarrow \kappa_{*},
\end{aligned}
$$

where $\lambda_{0}$ is identical to the scaling factor of the standard Efimov scenario, i.e., $\lambda_{0} \approx 22.694$. Since no general analytical solutions exist to the three-boson Schrödinger equation in the presence of spin-orbit coupling, we rely on numerics to support our claim. The claim that the 
discrete scaling symmetry survives in the presence of the spin-orbit-coupling terms can be understood intuitively by realizing that the spin-orbit-coupling terms modify the lowbut not the high-energy portions of the single-particle dispersion curves. To set the stage for the numerical calculations presented in the next section, we discuss a number of consequences of the discrete scaling symmetry.

The discrete scaling symmetry suggests a generalized radial scaling law for the three-boson system in the presence of 1D spin-orbit coupling in which the Efimov plot for $\vec{y}=\left(1 / a_{s}, K\right)^{T}$ discussed in the previous section is replaced by a generalized Efimov plot for

$\vec{y}=\left[1 / a_{s}, k_{\mathrm{so}}, \operatorname{sgn}(\Omega) \sqrt{m|\Omega| / \hbar^{2}}, \operatorname{sgn}(\tilde{\delta}) \sqrt{m|\tilde{\delta}| / \hbar^{2}}, K\right]^{T}$.

In the limit that the second, third, and fourth parameters vanish, each of the usual Efimov energies is fourfold degenerate due to the fact that the spin degrees of freedom enlarge the three-boson Hilbert space by a factor of 4 (from the $2^{3}=8$ independent spin configurations, one can construct four fully symmetric spin functions). For nonvanishing $k_{\mathrm{so}}, \Omega$, and $\tilde{\delta}$, we expect that the three-boson system supports four "unique" energy levels. Each of the four energies, collectively referred to as a manifold, is characterized by a vector $\vec{y}$. Knowing the dependence of each of these energy curves on $1 / a_{s}, k_{\mathrm{so}}, \operatorname{sgn}(\Omega) \sqrt{m|\Omega| / \hbar^{2}}$, and $\operatorname{sgn}(\tilde{\delta}) \sqrt{m|\tilde{\delta}| / \hbar^{2}}$, there should exist other energy manifolds for the same $\kappa_{*}$ that can be obtained from the manifold that has been mapped out without explicitly solving the three-boson Schrödinger equation again.

To see how, we switch from the five parameters given in Eq. (25) to the length $y=|\vec{y}|$ and four angles $\xi_{1}, \ldots, \xi_{4}$ for each of the four energy levels in the "reference manifold":

$$
\begin{gathered}
K=-y \sin \xi_{1} \sin \xi_{2} \sin \xi_{3} \sin \xi_{4}, \\
\operatorname{sgn}(\tilde{\delta}) \sqrt{m|\tilde{\delta}| / \hbar^{2}}=y \cos \xi_{1} \sin \xi_{2} \sin \xi_{3} \sin \xi_{4}, \\
\operatorname{sgn}(\Omega) \sqrt{m|\Omega| / \hbar^{2}}=y \cos \xi_{2} \sin \xi_{3} \sin \xi_{4}, \\
k_{\mathrm{so}}=y \cos \xi_{3} \sin \xi_{4},
\end{gathered}
$$

and

$$
1 / a_{s}=y \cos \xi_{4}
$$

The full range of possible $a_{s}, k_{\mathrm{so}}, \Omega$, and $\tilde{\delta}$ is covered if $\xi_{1}$, $\xi_{2}, \xi_{3}, \xi_{4} \in[0, \pi]$. The range of the angles is further constrained by the energy surfaces of the three-atom and atom-dimer thresholds (see Secs. IV and V). To obtain the $K$ for other manifolds, one chooses a direction of the vector $\vec{y}$ by fixing the angles $\xi_{1}$ to $\xi_{4}$ and reads off the values of the components of $\vec{y}$ for each of the four known energy levels. Using

$$
y=\sqrt{\left(a_{s}\right)^{-2}+\left(k_{\mathrm{so}}\right)^{2}+\frac{m|\Omega|}{\hbar^{2}}+\frac{m|\tilde{\delta}|}{\hbar^{2}}+K^{2}},
$$

it can be seen that the discrete transformation $a_{s} \rightarrow\left(\lambda_{0}\right)^{n} a_{s}$, $k_{\mathrm{so}} \rightarrow\left(\lambda_{0}\right)^{-n} k_{\mathrm{so}}, \Omega \rightarrow\left(\lambda_{0}\right)^{-2 n} \Omega, \tilde{\delta} \rightarrow\left(\lambda_{0}\right)^{-2 n} \tilde{\delta}, E \rightarrow\left(\lambda_{0}\right)^{-2 n} E$ implies $y \rightarrow\left(\lambda_{0}\right)^{-n} y$. Thus, dividing the "hyperradius" $y$ corresponding to the $j$ th energy in the reference manifold by $\left(\lambda_{0}\right)^{ \pm 1},\left(\lambda_{0}\right)^{ \pm 2}, \ldots$ and using the scaled value of $y$ in Eqs. (26)-(30), one obtains the values of the components of $\vec{y}$ for the $j$ th energy level in the other manifolds. The generalized scaling law is tested in the next section by considering two neighboring energy manifolds and confirming that the energy manifolds collapse onto each other if the discrete scaling transformation is applied to the energy levels in the more weakly bound manifold.

\section{NUMERICAL TEST OF THE GENERALIZED RADIAL SCALING LAW}

To facilitate the numerical calculations, we replace the two-body zero-range potential $V_{2 \mathrm{~b}, \mathrm{zr}}$ by an attractive Gaussian $V_{2 \mathrm{~b}, \mathrm{G}}$ with range $r_{0}$ and depth $v_{0}$,

$$
V_{2 \mathrm{~b}, \mathrm{G}}\left(r_{j k}\right)=v_{0} \exp \left(-\frac{r_{j k}^{2}}{2 r_{0}^{2}}\right),
$$

where $v_{0}$ is negative and adjusted such that $V_{2 \mathrm{~b}, \mathrm{G}}\left(r_{j k}\right)$ supports at most one two-body $s$-wave bound state. To reduce finite-range effects, we aim to work in the regime where the absolute value of the free-space $s$-wave scattering length $a_{s}$ is notably larger than $r_{0}$. Parameter combinations where the absolute value of the free-space $p$-wave scattering volume is large are excluded. The three-body zerorange potential $V_{3 \mathrm{~b}, \mathrm{zr}}$ is replaced by a repulsive Gaussian $V_{3 \mathrm{~b}, \mathrm{G}}$ with range $R_{0}$ and height $V_{0}$ :

$$
V_{V_{3 b, \mathrm{G}}}\left(r_{j k l}\right)=V_{0} \exp \left(-\frac{r_{j k l}^{2}}{2 R_{0}^{2}}\right) .
$$

In our numerical calculations, $R_{0}$ is fixed at $\sqrt{8} r_{0}$ and $V_{0}$ $\left(V_{0} \geq 0\right)$ is varied to dial in the desired three-body parameter $\kappa_{*}$. Specifically, we define $\kappa_{*}$ to be the binding momentum of the energetically lowest-lying universal three-body state at unitarity (infinite $a_{s}$ ) for $k_{\mathrm{so}}=\Omega=\tilde{\delta}=0$. Without the repulsive three-body potential, the lowest three-body state is not universal [52]. The repulsive three-body potential pushes the lowest three-body energy up and we adjust $V_{0}$, for fixed $v_{0}$ (infinite $a_{s}$ ), such that the energy of the lowest threebody state for finite $V_{0}$ is identical to the energy of the first excited three-body state for $V_{0}=0$. This corresponds to 
$\left(\kappa_{*}\right)^{-1} \approx 66.05 r_{0}$; i.e., the trimer is much larger than the intrinsic scales of the two- and three-body interactions. With the repulsive three-body potential turned on, the radial scaling law can be tested using the two lowest-lying energy manifolds.

We start the discussion of our numerical results by looking at the three-body spectrum for the standard Efimov scenario $\left(k_{\mathrm{so}}=\Omega=\tilde{\delta}=0\right)$. The reason for discussing this "reference system" is twofold: it illustrates how to check the validity of the radial scaling law for a case where it is known to hold and it gives us a sense for the finite-range corrections expected in the presence of spin-orbit coupling. The solid line in Fig. 1(b) shows the relative three-body energy of the energetically lowest-lying state as a function of the inverse of the $s$-wave scattering length for the Hamiltonian given in Eq. (5) with $V_{2 \mathrm{~b}, \mathrm{zr}}$ and $V_{3 \mathrm{~b}, \mathrm{zr}}$ replaced by $V_{2 \mathrm{~b}, \mathrm{G}}$ and $V_{3 \mathrm{~b}, \mathrm{G}}$, respectively. To "compress" the data, the horizontal and vertical axes employ a square-root and fourth-root representation. The scattering length is scaled by $r_{0}$ and the energy by $E_{\mathrm{sr}}$ :

$$
E_{\mathrm{sr}}=\frac{\hbar^{2}}{m r_{0}^{2}}
$$

The trimer energy merges with the three-atom threshold on the negative scattering length side at $r_{0} /\left|a_{s}\right| \approx 0.01$.

To get a feeling for the finite-range effects, we assume that the radial scaling law holds and apply it "in reverse." Specifically, using numerically determined pairs $\left(1 / a_{s}, K\right)$ corresponding to the excited state, the dots in Fig. 1(b) show the points $\left(\lambda_{0} r_{0} / a_{s}, \lambda_{0} r_{0} K\right)$, using - as for the lowest state-the square-root and fourth-root depiction. In the zero-range limit $\left(r_{0} \rightarrow 0\right.$ and $R_{0} \rightarrow 0$ ), the dots would lie on top of the solid line. The nearly perfect agreement between the solid line and the dots in Fig. 1(b) indicates that the finite-range effects are negligibly small for the parameter combinations considered.

To test the generalized radial scaling law proposed in Sec. III, we calculate the eigenenergies of states in the lowest and second-lowest manifolds of $\hat{\bar{H}}_{\text {rel }}$ (there are at most four states in each manifold) and scale the energies in the second-lowest manifold assuming that the generalized radial scaling law holds. If the energy curves collapse, the generalized radial scaling law is validated.

In the presence of the 1D spin-orbit coupling, the generalized Efimov plot has five axes. Clearly, visualizing energy surfaces that depend on four parameters is impossible and fully mapping out these high-dimensional dependences is computationally demanding. Thus, we consider selected cuts in the five-dimensional space. Our first cut uses $\left(k_{\mathrm{so}}\right)^{-1}=50 r_{0}, \Omega=2 E_{\mathrm{so}}=0.04 E_{\mathrm{sr}}$, where

$$
E_{\text {so }}=\frac{\left(\hbar k_{\mathrm{so}}\right)^{2}}{2 m}
$$

and $\tilde{\delta}=0$. For these parameters, we calculate the relative energy $E$ of the states in the lowest energy manifold. The solid lines in Fig. 2(a) show the quantity $-\mid\left(E-E_{\mathrm{th}}^{\text {aaa }}\right) /$ $\left.E_{\mathrm{sr}}\right|^{1 / 4}$ as a function of $\operatorname{sgn}\left(a_{s}\right)\left|r_{0} / a_{s}\right|^{1 / 2}$, where $E_{\mathrm{th}}^{\text {aaa }}$ denotes the energy of the lowest three-atom threshold whose wave function has the same total momentum $q_{3, z}$ along the $z$ axis as the three-body system. The determination of $E_{\mathrm{th}}^{\mathrm{aaa}}$ is discussed in Appendix C. The energy $E_{\mathrm{th}}^{\text {aaa }}$ is independent of $\kappa_{*}$ and referencing $E$ relative to the lowest three-atom threshold does not alter the generalized radial scaling law. Figure 2(a) shows that the lowest energy manifold consists of, depending on the value of $r_{0} / a_{s}$, zero, one, or two energy levels [the second and third excited
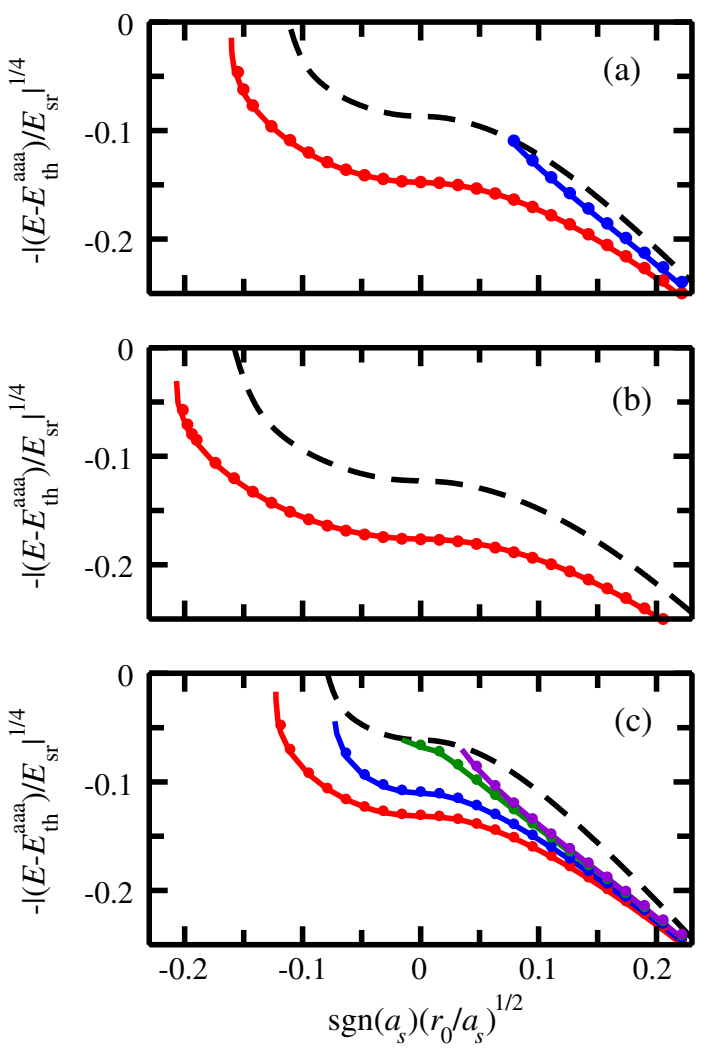

FIG. 2. Testing the generalized radial scaling law in the presence of 1D spin-orbit coupling. Panels (a)-(c) demonstrate the collapse of two neighboring energy manifolds for the finiterange interaction model $\left[\hat{\bar{H}}_{\text {rel }}\right.$ in Eq. (20) with $V_{2 \mathrm{~b}}=V_{2 \mathrm{~b}, \mathrm{G}}$ and $V_{3 \mathrm{~b}}=V_{3 \mathrm{~b}, \mathrm{G}} ; R_{0}=\sqrt{8} r_{0}$ and $\left(\kappa_{*}\right)^{-1} \approx 66.05 r_{0}$ ]. The energies of states in the lowest manifold (solid lines) are obtained for $\Omega=2 E_{\mathrm{so}}, \tilde{\delta}=0$, and (a) $\left(k_{\mathrm{so}}\right)^{-1}=50 r_{0},(\mathrm{~b})\left(k_{\mathrm{so}}\right)^{-1}=25 r_{0}$, and (c) $\left(k_{\mathrm{so}}\right)^{-1}=100 r_{0}$. In all three panels, the dashed lines show the atom-dimer threshold. The dots show the energies of states in the second-lowest manifold, with the generalized radial scaling law applied in reverse so as to collapse the three-body energies of states in the second-lowest manifold (dots) onto the three-body energies of the states in the lowest manifold (solid lines). For clarity, the scaled atom-dimer thresholds for the second-lowest energy manifold are not shown in any of the panels. 
states of the lowest manifold exist at larger $r_{0} / a_{s}$ than those shown in Fig. 2(a)].

The lowest three-body energy merges with the threeatom threshold on the negative $s$-wave scattering length side and with the atom-dimer threshold [dashed line in Fig. 2(a)] on the positive scattering length side. The determination of the atom-dimer threshold energy $E_{\mathrm{th}}^{\mathrm{ad}}$ is discussed in Appendix D. Just as the three-atom threshold, the atom-dimer threshold is independent of $\kappa_{*}$ and determined such that the momentum $q_{3, z}$ of the atom-dimer system is the same as that of the three-body system. The second-lowest state does not merge with the three-atom threshold on the negative $a_{s}$ side but with the atom-dimer threshold on the positive $a_{s}$ side.

Having determined the energies of the states in the lowest energy manifold, the next step is to calculate the energies of the states in the second-lowest energy manifold. To map the energies of the states in the second-lowest manifold onto the energies of the states in the lowest energy manifold, we use the same $r_{0}, R_{0}$, and $\kappa_{*}$ and calculate the energies of the states in the second-lowest energy manifold for a $k_{\mathrm{so}}$ that is $\lambda_{0}$ times smaller than the $k_{\mathrm{so}}$ used to calculate the energies of the states in the lowest energy manifold [i.e., for $\left(k_{\mathrm{so}}\right)^{-1} \approx 1,135 r_{0}$ ], for a $\Omega$ that is $\left(\lambda_{0}\right)^{2}$ times smaller than the $\Omega$ used to calculate the energies in the lowest energy manifold (i.e., for $\Omega \approx 7.77 \times 10^{-7} E_{\mathrm{sr}}$ ), and for $\tilde{\delta}=0$ (the scaling does not change zero) as a function of $r_{0} / a_{s}$. Having calculated the energies of the states in the second-lowest manifold for the scaled $k_{\mathrm{so}}, \Omega$, and $\tilde{\delta}$, the pairs $\left(1 / a_{s}, E-E_{\mathrm{th}}^{\text {aaa }}\right)$ are scaled (note that $E_{\mathrm{th}}^{\text {aaa }}$ for the excited state manifold is calculated using the scaled $k_{\mathrm{so}}, \Omega$, and $\tilde{\delta}$ values). The dots in Fig. 2(a) show the scaled pairs $\left[\lambda_{0} r_{0} / a_{s},-\left(\lambda_{0}\right)^{2}\left|E-E_{\mathrm{th}}^{\text {aaa }}\right| / E_{\mathrm{sr}}\right]$, using -as for the lowest energy manifold - the square-root and fourth-root depiction. It can be seen that the solid lines and dots agree very well. Note that the atom-dimer threshold for the second-lowest energy manifold also needs to be recalculated using the scaled $k_{\mathrm{so}}, \Omega$, and $\tilde{\delta}$ [the resulting energies lie essentially on top of the dashed line and are not shown in Fig. 2(a)]. The deviation between the solid line and dots is $0.025 \%$ for $\left(r_{0} / a_{s}\right)^{1 / 2}=0$ and $0.80 \%$ for $\left(r_{0} / a_{s}\right)^{1 / 2}=0.19$. These deviations are comparable to those between the corresponding atom-dimer thresholds [in this case, the deviations are $0.023 \%$ for $\left(r_{0} / a_{s}\right)^{1 / 2}=0$ and $0.82 \%$ for $\left(r_{0} / a_{s}\right)^{1 / 2}=0.19$ ]. We conclude that our numerical results are consistent with the generalized radial scaling law.

We emphasize that the scaling law has to be applied to all five axes of the generalized Efimov plot; i.e., to obtain the dots in Fig. 2(a) it is imperative to not only scale the two axes depicted but also the parameters corresponding to the three axes that are not depicted. The ratio of the lowest energy in neighboring manifolds at unitarity, e.g., is only equal to $22.694^{2}$ if the direction of $\hat{y}$ is the same for the two energy levels under consideration.
The energy scales $E_{\mathrm{so}},|\Omega|$, and $|\tilde{\delta}|$ are much smaller than $\left|E-E_{\mathrm{th}}^{\text {aaa }}\right|$ for a large portion of Fig. 2(a). The region close to the three-atom threshold is an exception. As such, it might be argued that the spin-orbit-coupling terms are too weak to notably influence the energy spectrum, possibly suggesting that the applicability of the generalized radial scaling law is trivial. One fact that speaks against this argumentation is that the shape of the energy levels is notably influenced by the spin-orbit-coupling terms. This is, e.g., reflected by the fact that the energy levels in a given energy manifold are not degenerate. To more explicitly demonstrate that the generalized radial scaling law holds when one or more of the energy scales associated with the spin-orbit-coupling terms are larger than the binding energy, we repeat the calculations for larger $k_{\mathrm{so}}$ than those used in Fig. 2(a). Specifically, to determine the energy of the lowest state in the lowest energy manifold [solid line in Fig. 2(b)], we use $\left(k_{\mathrm{so}}\right)^{-1}=25 r_{0}$ while keeping $r_{0}, R_{0}, \kappa_{*}$, and $\tilde{\delta}$ unchanged. The Raman coupling strength $\Omega$ is set to be equal to $2 E_{\mathrm{so}}$. To demonstrate the collapse of the energies of the lowest states in the second-lowest and lowest manifolds, we apply the generalized radial scaling law in the same way as in Fig. 2(a). The energy of the lowest state in the second-lowest manifold is shown by dots in Fig. 2(b). The agreement with the solid line is excellent, supporting our claim that the generalized radial scaling law is not limited to the case where the energy scales associated with the spin-orbit coupling are smaller than the binding energy of the trimer, provided these energies are notably smaller than $E_{\mathrm{sr}}$.

To show the characteristics of the excited states in the lowest manifold in more detail, we consider a smaller $k_{\mathrm{so}}$, $\left(k_{\mathrm{so}}\right)^{-1}=100 r_{0}$, and as before $\tilde{\delta}=0$ and $\Omega=2 E_{\mathrm{so}}$. The use of a smaller $k_{\text {so }}$ [solid lines in Fig. 2(c)] moves the merging points of the three-body energies corresponding to the excited states with the atom-dimer threshold to the left compared to Fig. 2(a). Again, scaling the parameters appropriately, the dots in Fig. 2(c) show the energies of the states in the second-lowest energy manifold. The dots agree nearly perfectly with the solid lines not only for the lowest state in the two manifolds but also for the excited states in the two manifolds, lending strong numerical support for the validity of the generalized radial scaling law and hence for the existence of the discrete scaling symmetry in the presence of 1D spin-orbit-coupling terms in the zero-range limit.

As already mentioned, the three-body parameter $\kappa_{*}$ for $V_{0}=0$, defined using the energy of the first excited state at unitarity in the absence of spin-orbit coupling, is identical to the $\kappa_{*}$ for the three-body interaction with finite $V_{0}$ used throughout this section. Turning on the spin-orbit coupling, we checked that the energies of states in the second-lowest manifold for $V_{0}=0$ agree well with the energies of states in the lowest manifold for the finite $V_{0}$. This provides evidence that the generalized radial scaling law is, just as 
the standard radial scaling law, independent of the details of the underlying microscopic interaction model. To confirm the continuous scaling symmetry of the three-body Hamiltonian in the presence of 1D spin-orbit coupling, we checked that the energies for different $\kappa_{*}$ can be mapped onto each other: If $\vec{y}$ describes a point on the Efimov plot for $\kappa_{*}$, then $\left(\kappa_{*}^{\text {new }} / \kappa_{*}\right) \vec{y}$ describes a point on the Efimov plot for the new $\kappa_{*}^{\text {new. }}$.

\section{EXPERIMENTAL IMPLICATIONS: ROLE OF CENTER-OF-MASS MOMENTUM}

Measuring signatures associated with two consecutive trimer energy levels is challenging, especially for equalmass bosons, due to the relatively large discrete scaling factor of 22.694. The reason is that the absolute value of the scattering length should, on the one hand, be notably larger than the van der Waals length $r_{\mathrm{vdW}}$ and, on the other hand, be smaller than the de Broglie wave length $\lambda_{\mathrm{dB}}[8,11]$. Despite these challenges, the discrete scaling symmetry underlying the standard Efimov scenario has been confirmed experimentally by monitoring the atom losses of an ultracold thermal gas of Cs atoms as a function of the $s$-wave scattering length [20] (for unequal mass mixtures, see Refs. [53,54]). When the trimer energy is degenerate with the three-atom threshold [dots in Fig. 1(a); the corresponding critical scattering lengths are denoted by $a_{-}^{(n)}$ ] or with the atom-dimer threshold [squares in Fig. 1(a); the corresponding critical scattering lengths are denoted by $\left.a_{*}^{(n)}\right]$, the losses are enhanced. Since the critical scattering lengths for consecutive trimer states are related to the scaling factor $\lambda_{0}$, these atom-loss measurements provide a direct confirmation of the discrete scaling symmetry. In addition, other characteristics of the standard Efimov scenario have been measured [8,11-13]. For example, the critical scattering lengths $a_{-}^{(n)}$ and $a_{*}^{(n)}$ for a given trimer level $n$ are related to each other by a universal number. Correspondingly, the experimentally determined ratio $a_{-}^{(n)} / a_{*}^{(n)}$ can be viewed as a test of the functional form of the energy levels shown in Fig. 1(a). Other experimental tests of the standard Efimov scenario include the determination of the binding energy of an Efimov trimer via radio frequency spectroscopy $[17,18]$, the imaging of the quantum mechanical density of the helium Efimov trimer via Coulomb explosion [21], and the observation of four- and five-body loss features that are universally linked to the critical scattering lengths of the Efimov trimer [55-58].

Directly measuring the discrete scaling symmetry in the presence of spin-orbit coupling requires varying the inverse of the $s$-wave scattering length by the scaling factor $\lambda_{0}$, as in the standard Efimov scenario, as well as varying the spinorbit-coupling parameters $\Omega, E_{\mathrm{so}}$, and $\tilde{\delta}$ by $\left(\lambda_{0}\right)^{2}$. Covering such a wide range of parameters is expected to be very challenging experimentally. In what follows we instead focus on the situation where the spin-orbit-coupling parameters $k_{\mathrm{so}}$ and $\Omega$ are held fixed while the $s$-wave scattering length $a_{s}$ and generalized detuning $\tilde{\delta}$ are varied. An analogous study for the standard Efimov scenario would look at the three-boson system for a fixed finite $s$-wave scattering length. In this case, the energy spacing would not be $\left(\lambda_{0}\right)^{2}$; however, the energies of neighboring states would still be uniquely related to each other.

For concreteness, we consider the ${ }^{133} \mathrm{Cs}$ system [59], for which the three-atom resonances in the absence of spinorbit coupling occur at the critical scattering lengths $a_{-}^{(0)} \approx$ $-936 a_{0}$ and $a_{-}^{(1)} \approx-20,190 a_{0}$ [20]. Here, $a_{0}$ denotes the Bohr radius and the superscripts "(0)" and "(1)" indicate that these critical scattering lengths are for the ground and first excited Efimov trimers, respectively. Applying our numerical result $\kappa_{*} a_{-}=-1.505$ to the first excited state, the Cs system is characterized by $\left(\kappa_{*}\right)^{-1} \approx 13,416 a_{0}$.

Figure 3 shows the negative of the binding energy of the lowest state in the first excited manifold for $k_{\mathrm{so}} / \kappa_{*} \approx 1.32$ and $\Omega=2 E_{\mathrm{so}}$ as functions of the inverse of the $s$-wave scattering length and the generalized detuning $\tilde{\delta}$ using, as in the previous sections, that the scattering lengths are the same for all spin channels. Using Cs's $a_{-}^{(1)}$, these parameters correspond to $\left(k_{\mathrm{so}}\right)^{-1} \approx 10,156 a_{0}, E_{\mathrm{so}} / h \approx 0.132 \mathrm{kHz}$, and $\Omega / h \approx 0.264 \mathrm{kHz}$. Comparison with the ${ }^{87} \mathrm{Rb}$ experiment at NIST [28], which uses $\left(k_{\mathrm{so}}\right)^{-1} \approx 3,410 a_{0}$ (corresponding to $E_{\mathrm{so}} / h \approx 1.786 \mathrm{kHz}$ ) and $\Omega / h$ values ranging from 0 to about $10 \mathrm{kHz}$, suggests that the parameter regime covered in Fig. 3 is reasonable. Figure 3 shows that the three-boson binding energy for a fixed scattering length is largest for $\tilde{\delta}=0$ (this is where the three-atom threshold has a degeneracy of six; see Appendix C). In addition, there exists an enhancement of the

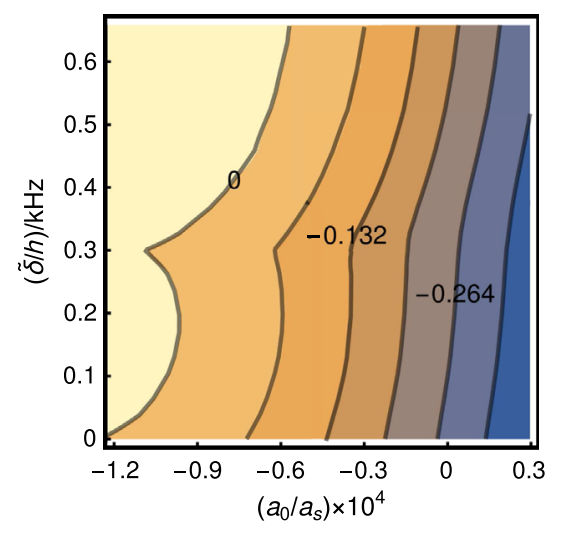

FIG. 3. The contours show the negative of the three-boson binding energy, in $\mathrm{kHz}$, of the lowest state in the second-lowest manifold as functions of $\left(a_{s}\right)^{-1}$ and $\tilde{\delta}$ for $k_{\mathrm{so}} / \kappa_{*} \approx 1.32$ and $\Omega=2 E_{\mathrm{so}}$, where $\kappa_{*}$ denotes the binding momentum of the first excited Efimov trimer at unitarity in the absence of spin-orbit coupling. The conversion to $a_{0}$ and $\mathrm{kHz}$ is done using the experimentally determined value of $a_{-}^{(1)}$ for Cs (see text). The calculations are performed for $\hat{\bar{H}}_{\text {rel }}$ with $V_{2 \mathrm{~b}}=V_{2 \mathrm{~b}, \mathrm{G}}$ and $V_{3 \mathrm{~b}}=0\left[\left(\kappa_{*}\right)^{-1} \approx 66.05 r_{0}\right]$. 
binding for $\tilde{\delta} / h \approx 0.301 \mathrm{kHz}$ (this is where the three-atom threshold has a degeneracy of four; see Appendix C). As $\tilde{\delta}$ goes to infinity, the trimer in the presence of the 1D spin-orbit coupling becomes unbound at the same scattering length as the corresponding trimer in the absence of spin-orbit coupling (i.e., at $a_{s} \approx-20,190 a_{0}$ ).

The three-boson binding energy shown in Fig. 3 is calculated by enforcing that the three-boson threshold has the same center-of-mass momentum as the trimers (see Appendixes $\mathrm{C}-\mathrm{E}$ ). If the detuning $\delta$ is equal to zero, the generalized detuning $\tilde{\delta}$ is directly proportional to the $z$ component $q_{3, z}$ of the center-of-mass momentum [see Eq. (21)]. In this case, the trimer is bound maximally for $q_{3, z}=0$. However, for finite detuning $\delta$, the most strongly bound trimer has a finite center-of-mass momentum. A similar dependence on the center-of-mass momentum was pointed out in Refs. $[35,37,61]$ for the twofermion system.

The dependence of $\hat{\bar{H}}_{\text {rel }}$ on $q_{N=3, z}$ is a key characteristic of systems with 1D spin-orbit coupling. A similar dependence exists for three-body systems in the presence of 2D or 3D spin-orbit coupling (in these cases, the relative Hamiltonian depends on two or all three components of $\vec{q}_{N=3}$ ) and for three-body systems on a lattice (in this case, $\vec{q}_{N=3}$ is a lattice or quasimomentum vector). In all works known to us [16,46-48], the assumption $\vec{q}_{N=3}=0$ is made prior to obtaining concrete results. Table I contrasts studies for systems, which possess a center-of-mass momentum dependence, with the "standard" three-boson Efimov system (first row), for which the relative Hamiltonian is independent of $\vec{q}_{N=3}$. In the standard Efimov case, the lowest atom-dimer threshold of the relative Hamiltonian is given by the energy $E_{a}$ of an atom with vanishing atom momentum vector $\vec{q}_{a}$ ( $E_{a}$ is equal to zero) plus the energy $E_{d}\left(\vec{q}_{d}=0\right)$ of a dimer with vanishing dimer momentum vector $\vec{q}_{d}$. When the relative Hamiltonian depends on $\vec{q}_{N=3}$, the atom-dimer threshold needs to be determined carefully, since the trimer with fixed $\vec{q}_{N=3}$ can break up into an atom with finite momentum and into a dimer with finite momentum in such a way that the generalized three-body center-of-mass momentum is conserved. Of the many break-up configurations that conserve the three-body center-of-mass momentum, the one with the lowest energy defines the atom-dimer threshold. Table I shows that the definition of the lowest atom-dimer threshold of the relative Hamiltonian varies in the literature. The definitions employed in Refs. [16,47] disagree with the definition used in the present work (last row of Table I). While the definition of Ref. [47] may be meaningful in a many-body context (see also Ref. [61]), we fail to see how the definition of Ref. [16] can, in general, be correct.

It is proposed that the center-of-mass momentum dependence can be observed experimentally by performing atom-loss measurements for fixed $k_{\mathrm{so}}, \Omega$, and $\delta$ on a cold thermal atomic gas. Tuning the $s$-wave scattering length, one expects-just as in the case where the spin-orbit coupling is absent-enhanced losses when the trimer energy is degenerate with the three-atom threshold. However, in contrast to the standard Efimov scenario, such a degeneracy exists for a range of scattering lengths provided the trimers embedded in the thermal gas have different three-body center-of-mass momenta (the exact distribution of center-of-mass momenta is set by the temperature of the gas sample). Figure 3 shows that the critical scattering length $a_{-}^{(1)}$ changes, for the Cs example, from $-20190 a_{0}$ for large $\tilde{\delta}$ to $-7791 a_{0}$ for $\tilde{\delta}=0$. Provided the three-body center-of-mass momenta are spread over the range covered on the vertical axis in Fig. 3, one expects enhanced losses over the entire scattering length window. The difference between the losses in the presence and absence of the spin-orbit-coupling terms can be interpreted as a few-body probe of the breaking of the Galilean invariance in the presence of spin-orbit coupling.

An important question is whether the changes of the loss features related to the lowest state in the second-lowest manifold will be washed out by finite temperature effects. A comprehensive answer to this question will require performing three-body recombination calculations, which include thermal averaging, in the presence of spin-orbit coupling. Such calculations are beyond the scope of this work. Given that the energy scales associated with the spinorbit coupling are, for the example considered in Fig. 3,

TABLE I. Summary of three-particle studies. The standard Efimov scenario (first row) is contrasted with three-particle systems for which the relative Hamiltonian $\hat{\bar{H}}_{\text {rel }}$ depends parametrically on the generalized three-body center-of-mass momentum vector $\vec{q}_{N=3}$. The last column lists the atom-dimer threshold of the relative Hamiltonian. The symbols $E_{d}, E_{a}, \vec{q}_{d}$, and $\vec{q}_{a}$ denote the energy of the dimer, energy of the atom, generalized momentum of the dimer, and generalized momentum of the atom, respectively. SOC, spin-orbit coupling; $F$, fermion; $X$, a particle different from $F ; B$, boson.

\begin{tabular}{|c|c|c|c|}
\hline System & $\hat{\bar{H}}_{\text {rel }}=\hat{\bar{H}}_{\text {rel }}\left(\vec{q}_{N=3}\right) ?$ & Restriction? & Atom-dimer threshold (rel. Ham.) \\
\hline Three s & no & no & $=0)+E_{a}\left(\vec{q}_{a}\right.$ \\
\hline$F F X ; X$ feels 2D SOC; Borromean binding [47] & yes & $\vec{q}_{N=3}=0$ & $\min _{\vec{q}_{d}} E_{d}\left(\vec{q}_{d}\right)+\min _{\vec{q}_{a}} E_{a}\left(\vec{q}_{a}\right)$ \\
\hline$F F X ; X$ feels 3D SOC; universal or Efimov trimers $[46,48]$ & yes & $\vec{q}_{N=3}=0$ & $\min _{\vec{q}_{d}+\vec{q}_{a}=\vec{q}_{N=3}}\left[E_{d}\left(\vec{q}_{d}\right)+E_{a}\left(\vec{q}_{a}\right)\right]$ \\
\hline$B B B$ quasiparticles on lattice; Efimov trimers [16] & yes & $\vec{q}_{N=3}=0$ & $E_{d}\left(\vec{q}_{d}=0\right)+E_{a}\left(\vec{q}_{a}=0\right)$ \\
\hline$B B B ; B$ 's feel 1D SOC (this work) & yes & no & $\min _{\vec{q}_{d}+\vec{q}_{a}=\vec{q}_{N=3}}\left[E_{d}\left(\vec{q}_{d}\right)+E_{a}\left(\vec{q}_{a}\right)\right]$ \\
\hline
\end{tabular}


comparable to $\hbar^{2} \kappa_{*}^{2} / m$ and that the binding energy of the trimer near the three-atom threshold is much smaller than $\hbar^{2} \kappa_{*}^{2} / m$, we are hopeful that the temperatures realized in previous Cs experiments ( $T \geq 7.7 \mathrm{nK}$ ) [20] are low enough to observe the impact of the spin-orbit-coupling terms on the loss features. For example, without spin-orbit coupling, the loss coefficient $L_{3}$ is maximal around $-20000 a_{0}$ and reaches about half of its maximum value at around $-10000 a_{0}$ [see Fig. 1(a) of Ref. [20]]. In the presence of spin-orbit coupling, the loss feature is expected to be centered over the range $-20190 a_{0}$ to $-7790 a_{0}$, leading to an observable modification of the shoulder on the less negative scattering length side. The shape of the shoulder is expected to carry a signature of the nonmonotonic dependence of the critical scattering length $a_{-}^{(1)}$ on the center-ofmass momentum. For example, three different $\tilde{\delta}$ correspond to the same $a_{-}^{(1)}$ for $a_{-}^{(1)} \in\left[-10330 a_{0},-9160 a_{0}\right]$, but each $\tilde{\delta}$ corresponds to a unique $a_{-}^{(1)}$ for $a_{-}^{(1)} \in$ $\left[-20190 a_{0},-10330 a_{0}\right]$ and $a_{-}^{(1)} \in\left[-9160 a_{0},-7790 a_{0}\right]$.

For the same spin-orbit-coupling parameters, the critical scattering length $a_{-}^{(0)}$, associated with the lowest state in the lowest manifold, displays essentially no dependence on $\tilde{\delta}$; i.e., the associated three-atom loss feature should only be minimally affected by the spin-orbit-coupling terms. Intuitively, this can be understood by realizing that the energy scales associated with the spin-orbit-coupling parameters are much smaller than the binding energy of the lowest-lying trimer state. The fact that the loss features for the lowest state in the lowest and second-lowest manifolds are expected to be very different can also be understood from the generalized radial scaling law. Fixing the spin-orbit-coupling parameters corresponds to looking at particular cuts in the five-dimensional parameter space as opposed to looking along a specific radial direction. As a consequence, the loss features for the two manifolds can be very different even if the scattering lengths at which the loss features occur are, roughly, spaced by $\lambda_{0}$.

\section{CONCLUDING REMARKS}

This work analyzes what happens to the three-boson Efimov spectrum if 1D spin-orbit-coupling terms, realizable in cold atoms as well as in photonic crystals and mechanical setups, are added to the Hamiltonian. The spinorbit-coupling terms introduce a parametric dependence of the relative Hamiltonian on the center-of-mass momentum vector. A similar center-of-mass momentum vector dependence exists for few-body systems with short-range interactions on a lattice. The present work maps out, for the first time, the three-boson spectrum as a function of the centerof-mass momentum vector. It is found that the three-boson system in the presence of 1D spin-orbit coupling obeys a generalized radial scaling law in a five-dimensional parameter space, which is associated with a discrete scaling symmetry. Within the framework of effective field theory, the existence of the discrete scaling symmetry can be rationalized by scale separation: The discrete scaling symmetry of the standard Efimov scenario "survives" provided the additional length scales are much larger than the ranges of the intrinsic interactions. While our work focuses on 1D spin-orbit coupling, the discrete scaling symmetry should persist for other types of spin-obitcoupling schemes as well.

The spin degrees of freedom lead-for the type of spinorbit coupling considered in this work - to a quadrupling of each Efimov trimer (manifold of four states). The threebody states in a given manifold are tied to one of the three two-boson states [62]. The point $\left(1 / a_{s}, k_{\mathrm{so}}, \Omega, \tilde{\delta}\right)=$ $(0,0,0,0)$ serves as an accumulation point for all four states of the manifold; i.e., in its vicinity, there exist infinitely many three-body bound states. The rich structure of two- and three-boson states should be amenable to experimental verification. Because of the dependence of the trimers on the center-of-mass momentum, the scattering length at which the lowest trimer in the second-lowest manifold merges with the atom-atom-atom threshold is, in fact, a scattering length window. Similar scattering length windows exist for the excited states in the second-lowest manifold. It was argued that these scattering length windows should be observable in cold-atom loss experiments, providing a direct few-body signature of the breaking of the Galilean invariance of systems with spin-orbit coupling.

If one considers a cut in the generalized five-dimensional Efimov plot, energy levels are not spaced by the scaling factor $\left(\lambda_{0}\right)^{2}$. Let us consider the situation where $a_{s}$ is infinitely large and where $k_{\mathrm{so}}, \Omega$, and $\tilde{\delta}$ are finite. In this case, the low-energy scales associated with the 1D spinorbit-coupling terms lead to a cutoff of the hyperradial $-1 / R^{2}$ Efimov potential curve ( $R$ denotes the three-body hyperradius). As a consequence, the number of three-body bound states at unitarity is not infinitely large. Albeit due to a different mechanism, this is similar in spirit to the disappearance of Efimov states if an Efimov trimer is placed into a gas of bosons or fermions $[63,64]$. This is also similar in spirit to a rather different system, namely the $\mathrm{H}^{-}$ ion. Taking only Coulomb interactions into account, one obtains a $-1 / r^{2}$ attraction [65], where $r$ is the distance between the extra electron and the atom. Relativistic effects introduce an additional length scale, which renders the number of bound states finite [65].

The calculations in this work are performed assuming that the interactions between the different spin channels are all equal. If one of the scattering lengths is large and tunable while the others are close to zero, the discrete scaling symmetry should still hold (approximately). To find the functional form of the energies for this scenario, the spectrum has to be recalculated.

The study we present should be viewed as a first step toward uncovering the rich three- and higher-body physics that emerges as a consequence of the unique coupling 
between the relative and center-of-mass degrees of freedom in cold-atom systems in the presence of artificial gauge fields. While somewhat different in nature, the coupling of these degrees of freedom in the relativistic Klein-Gordon and Dirac equations and quantum field theories has captured physicists' imagination for many decades.

\section{ACKNOWLEDGMENTS}

Support by the National Science Foundation through Grants No. PHY-1509892 and No. PHY-1745142 is gratefully acknowledged. This work used the Extreme Science and Engineering Discovery Environment (XSEDE), which is supported by NSF Grant No. OCI-1053575. Some of the computing for this project was performed at the OU Supercomputing Center for Education and Research (OSCER) at the University of Oklahoma (OU).

\section{APPENDIX A: JACOBI COORDINATES}

The single-particle and Jacobi coordinates employed in this work are related through the matrix $\underline{U}$ [66],

$$
\left(\vec{\rho}_{1}, \ldots, \vec{\rho}_{N}\right)^{T}=\underline{U}\left(\vec{r}_{1}, \ldots, \vec{r}_{N}\right)^{T}
$$

where

$$
\underline{U}=\left(\begin{array}{cc}
1 & -1 \\
1 / 2 & 1 / 2
\end{array}\right)
$$

for the equal-mass two-particle system and

$$
\underline{U}=\left(\begin{array}{ccc}
1 & -1 & 0 \\
1 / 2 & 1 / 2 & -1 \\
1 / 3 & 1 / 3 & 1 / 3
\end{array}\right)
$$

for the equal-mass three-particle system. The transformation matrix $\underline{U}$ also defines the matrices $\hat{\Sigma}_{j, z}$ $(j=1, \ldots, N-1)$. For the two-body system, we have

$$
\hat{\Sigma}_{1, z}=\hat{\sigma}_{1, z} \otimes I_{2}-I_{1} \otimes \hat{\sigma}_{2, z}
$$

For the three-body system, we have

$$
\hat{\Sigma}_{1, z}=\hat{\sigma}_{1, z} \otimes I_{2} \otimes I_{3}-I_{1} \otimes \hat{\sigma}_{2, z} \otimes I_{3}
$$

and

$$
\begin{aligned}
\hat{\Sigma}_{2, z}= & \frac{1}{2}\left(\hat{\sigma}_{1, z} \otimes I_{2} \otimes I_{3}+I_{1} \otimes \hat{\sigma}_{2, z} \otimes I_{3}\right) \\
& -I_{1} \otimes I_{2} \otimes \hat{\sigma}_{3, z} .
\end{aligned}
$$

\section{APPENDIX B: EXPLICITLY CORRELATED BASIS SET EXPANSION APPROACH}

This appendix discusses our approach to solving the fewparticle time-independent Schrödinger equation using a basis set expansion in terms of explicitly correlated Gaussian basis functions, which contain nonlinear variational parameters that are optimized semistochastically.

As we discuss in the main text, we are interested in bound states, i.e., eigenstates that approach zero at large interparticle distances. To solve the time-independent Schrödinger equation, we expand the relative portion $\Phi_{\text {rel }}$ of the eigenstate $\Psi$ sought in terms of a set of nonorthogonal eigenfunctions $\psi_{j}[66,67]$ :

$$
\Phi_{\mathrm{rel}}=\sum_{j=1}^{N_{b}} c_{j} \psi_{j}
$$

The basis functions $\psi_{j}$ depend on the relative spatial and the spin degrees of freedom,

$$
\psi_{j}=\hat{\mathcal{S}}\left(\phi_{j}\left(\vec{\rho}_{1}, \ldots, \vec{\rho}_{N-1}\right) \chi_{j}\right),
$$

where $\chi_{j}$ denotes an $N$-particle spin function that is chosen from the complete set of $2^{N}$ possible spin functions. The spatial parts $\phi_{j}$ are written in terms of a total of $(N-1)(N / 2+3)$ nonlinear variational parameters $d_{k l}^{(j)}$ and $\vec{s}_{k}^{(j)}$ :

$$
\phi_{j}=\exp \left(-\sum_{k<l}^{N} \frac{r_{k l}^{2}}{2 d_{k l}^{(j)}}+\sum_{k=1}^{N-1} i \vec{s}_{k}^{(j)} \cdot \vec{\rho}_{k}\right) .
$$

Here, the superscript " $(j)$ " serves to remind us that each basis function is characterized by a set of nonlinear variational parameters. The nonlinear variational parameters $d_{k l}^{(j)}$ determine the widths of the Gaussian factors of the basis functions. These widths are governed, roughly, by the two-body interaction terms in the Hamiltonian. The nonlinear parameters $\vec{s}_{k}^{(j)}$ determine the spatial oscillations due to the $k_{\mathrm{so}}$-dependent one-body terms. We do find that the values of $\vec{s}_{k}^{(j)}$ can depend quite strongly on the spin basis function considered. This shows that the parameters $\vec{s}_{k}^{(j)}$ govern, to leading order, the interplay between the spatial and spin degrees of freedom. Of course, strictly speaking, the influence of the single-particle and two-particle interaction terms in the Hamiltonian on the eigenstates cannot be separated; rather, the eigenstates are the result of the relative importance of each of these terms and the kinetic energy terms. The $N(N-1) / 2$ nonlinear parameters $d_{k l}^{(j)}$ and the $3(N-1)$ nonlinear parameters contained in $\vec{s}_{k}^{(j)}$ are optimized semistochastically; i.e., the basis set is constructed so as to minimize the energy of the eigenstate 
under study. The symmetrizer $\hat{\mathcal{S}}$ in Eq. (B2) ensures that the basis functions, and hence the eigenstate sought, are fully symmetrized. For the two-boson system, e.g., the symmetrizer reads $\hat{\mathcal{S}}=\left(1+\hat{P}_{12}\right) / \sqrt{2}$, where $\hat{P}_{12}$ exchanges the spatial and spin degrees of freedom of particles 1 and 2 . For $N$ identical particles, the symmetrizer contains $N$ ! terms.

The linear parameters $c_{j}$ are determined by solving the generalized eigenvalue problem that depends on the Hamiltonian matrix and, due to the nonorthogonality of the basis functions, the overlap matrix [66]. The results presented in this paper utilize basis sets consisting of up to $N_{b}=1800$ basis functions. The key strengths of the numerical approach employed are that the Hamiltonian and overlap matrix elements have compact analytical expressions and that the nonlinear variational parameters can be adjusted so as to capture correlations that occur on scales smaller than $r_{0}$ and larger than $\left(k_{\mathrm{so}}\right)^{-1}$.

The energies for the $N=2$ system depend on the parameters $a_{s}, k_{\mathrm{so}}, \Omega$, and $\tilde{\delta}$. For $N=3$, they additionally depend on $\kappa_{*}$. In practice, we set $\vec{q}_{N}=0$ and scan $\Omega / E_{\mathrm{so}}$, $\delta / E_{\mathrm{so}}$, and $a_{s} k_{\mathrm{so}}$ (for $N=3$, we fix $\kappa_{*}$ ). To obtain the relative eigenenergies $E$ and eigenstates $\Phi_{\text {rel }}$ for finite $q_{N, z}$, we do not need to redo the numerical calculations. Instead, we use the "conversion" implied by Eq. (21); i.e., the relative eigenenergy and eigenstates are obtained by changing from $\delta$ to $\tilde{\delta}$. The full eigenstates $\Psi$ are obtained by multiplying the $\vec{q}_{N}=0$ eigenstates by the center-ofmass piece $\Phi_{\text {c.m. }}$.

As in the case without spin-orbit coupling [68], it tends to be more efficient to describe each relative eigenstate $\Phi_{\text {rel }}$ by its own basis set as opposed to constructing one basis set that describes multiple eigenstates well. We refer to the eigenstate sought - this could be the ground state or one of the excited states-as the target state. To construct the basis, we add one basis function at a time. Let us assume that we have a basis set of size $N_{\text {initial }}$ and that we want to enlarge the basis set by one basis function. To do this, we generate $N_{\text {trial }}$ trial basis functions ( $N_{\text {trial }}$ is of the order of a few hundred to a few thousand) and calculate the energy $E_{k}$ $\left(k=1, \ldots, N_{\text {trial }}\right)$ of the target state for each of these enlarged basis sets. Assuming that the generalized eigenvalue problem for the basis set of size $N_{\text {initial }}$ has been solved, the trial energies can be calculated via a rootfinding procedure [66], which is, generally, computationally significantly faster than solving the generalized eigenvalue problem. The basis function to be added is determined by which of the $N_{\text {trial }}$ trial energies is lowest, i.e., by looking for the trial basis function that lowers the energy of the target state the most. After the "best" trial function has been added, the generalized eigenvalue problem is solved for the basis set of size $N_{\text {initial }}+1$ and the procedure is repeated to generate a basis set of size $N_{\text {initial }}+2$.

The convergence of the energy with increasing basis set size can be improved significantly by choosing "good" basis functions, i.e., by generating basis functions that efficiently cover the entire Hilbert space. Conversely, if the basis set is not constructed carefully, the energy may not even converge. In our implementation, the parameters $d_{k l}^{(j)}$ and $\vec{s}_{k}^{(j)}$ that characterize the spatial part of the basis functions are chosen from carefully adjusted parameter windows. For example, the $d_{k l}^{(j)}$ are chosen so as to cover length scales ranging from less than $r_{0}$ to a few times the length set by the binding energy of the target state. Since the target energy is, in general, not known a priori, the parameter windows are typically refined based on results obtained in preliminary calculations. To choose parameter windows for the $x, y$ and $z$ components of $\vec{s}_{k}^{(j)}$, we are guided by the noninteracting two- and three-particle dispersion curves. For example, if the noninteracting two-particle dispersion along the $z$ coordinate exhibits two minima, we choose $s_{1, z}^{(j)}$ uniformly from the windows $\left[-s_{\max , z},-s_{\min , z}\right]$ and $\left[s_{\min , z}, s_{\max , z}\right]$, with the windows including the momenta at which the dispersion curve is minimal. The parameters $s_{1, x}^{(j)}$ and $s_{1, y}^{(j)}$ are selected from windows that include zero. The widths of the parameter windows are adjusted through an educated trial and error procedure. Among other things, we check if the parameters selected by the code are clumped in a particular region of the parameter space.

\section{APPENDIX C: THREE-ATOM THRESHOLD}

The three-atom system with center-of-mass momentum $\vec{q}_{N}, N=3$, is bound if its energy is lower than that of three infinitely far separated atoms with the same center-of-mass momentum and, if a two-body bound state exists, lower than that of an infinitely far separated dimer and atom with the same center-of-mass momentum. To determine the lowest three-body scattering threshold, one thus needs to know the lowest dimer binding energy for all two-body center-of-mass momenta. As a consequence, the three-body scattering threshold depends on $\tilde{\delta} / E_{\mathrm{so}}$ and $\Omega / E_{\mathrm{so}}$ as well as on $a_{s} k_{\text {so }}$. We define the scattering threshold $E_{\text {th }}$ using $\hat{\bar{H}}_{\text {rel }}$ and denote the eigenenergies of $\hat{\bar{H}}_{\text {rel }}$ by $E$.

We start by determining the lowest relative three-body scattering threshold in the absence of two-body bound states. In this case, the lowest relative scattering threshold is determined by the minimum energy of the noninteracting relative dispersion curves for fixed $\tilde{\delta} / E_{\mathrm{so}}$ and $\Omega / E_{\mathrm{so}}$. The dispersion curves depend on two relative momenta (namely, $q_{1, z}$ and $q_{2, z}$ ), and the total number of dispersion curves is eight. Figures 4(a)-4(c) are for the uncoupled case $(\Omega=0)$ and $\tilde{\delta} / E_{\mathrm{so}}=0,8 / 3$, and 3.5 , respectively. The number of global minima changes from six for $\tilde{\delta}=0$ [see Fig. 4(a)] to three for $0<\tilde{\delta} / E_{\mathrm{so}}<8 / 3$ (not shown) to four for $\tilde{\delta} / E_{\mathrm{so}}=8 / 3$ [see Fig. 4(b)] to one for $\tilde{\delta} / E_{\mathrm{so}}>8 / 3$ [see Fig. 4(c)]. A finite Raman coupling strength $\Omega$ introduces a 

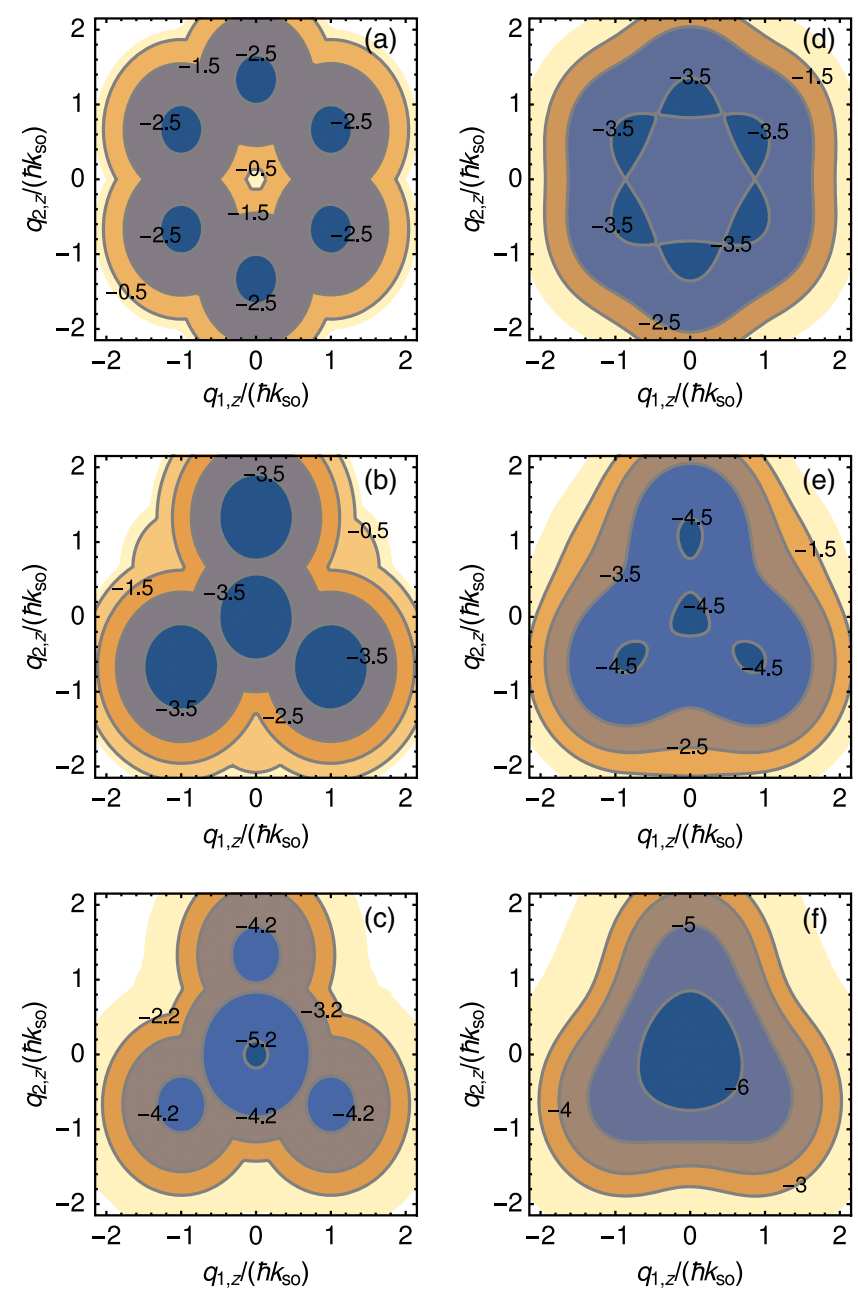

FIG. 4. Lowest noninteracting relative three-atom dispersion curve. The contours show the lowest noninteracting relative three-atom dispersion curve, in units of $E_{\mathrm{so}}$, as functions of $q_{1, z}$ and $q_{2, z}$. (a)-(c) $\Omega / E_{\mathrm{so}}=0$ and $\tilde{\delta} / E_{\mathrm{so}}=0,8 / 3$, and 3.5, respectively. (d)-(f) $\Omega / E_{\mathrm{so}}=2$ and $\tilde{\delta} / E_{\mathrm{so}}=0,2.278$, and 4 , respectively.

coupling between the different spin channels. As an example, Figs. 4(d)-4(f) show the lowest relative noninteracting dispersion curves for $\Omega / E_{\mathrm{so}}=2$ and $\tilde{\delta} / E_{\mathrm{so}}=0$, 2.278, and 4, respectively. As for vanishing Raman coupling, the number of global minima changes from six to three (not shown) to four to one with increasing $\tilde{\delta}$. However, the critical generalized detuning $\tilde{\delta}$ at which these changes occur differs for $\Omega / E_{\mathrm{so}}=2$ and $\Omega=0$.

The minimum of the noninteracting relative three-atom dispersion curves defines, assuming two-body bound states are absent, the lowest three-atom scattering threshold. Figure 5 shows the lowest three-atom scattering threshold energy $E_{\text {th }}^{\text {aaa }}$ as functions of $\tilde{\delta} / E_{\mathrm{so}}$ and $\Omega / E_{\mathrm{so}}$. The thick open circles and thick dashed line indicate the parameter combinations at which the number of global minima is six and four, respectively. For parameter combinations above the thick open circles and below the thick dashed line, the

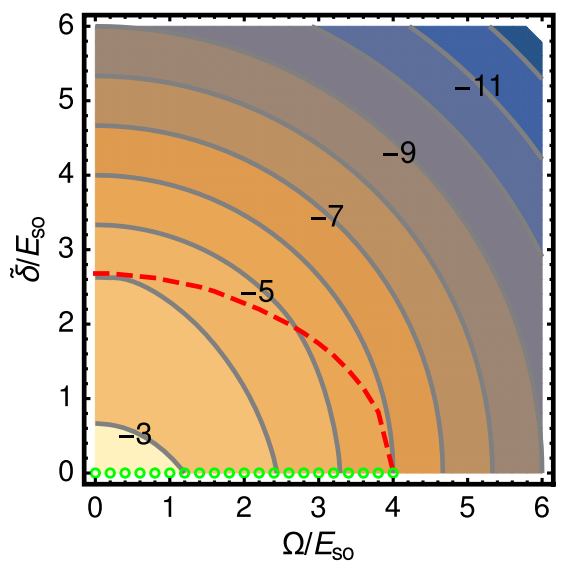

FIG. 5. Lowest relative three-atom scattering threshold. The contours show the energy $E_{\mathrm{th}}^{\mathrm{aaa}}$, in units of $E_{\mathrm{so}}$, of the lowest threeatom scattering threshold as functions of $\Omega / E_{\mathrm{so}}$ and $\tilde{\delta} / E_{\mathrm{so}}$. The thick open circles and thick dashed line indicate the parameter combinations $\left(\tilde{\delta} / E_{\mathrm{so}}, \Omega / E_{\mathrm{so}}\right)$ at which the degeneracy of the lowest three-atom scattering threshold is six and four, respectively.

number of global minima of the lowest noninteracting relative dispersion curve is equal to three. Above the thick dashed line the number of global minima is equal to one. If we assume that the three-body binding energy is, approximately, largest when the degeneracy of the lowest noninteracting relative dispersion curve is largest, then Fig. 5 suggests that the three-body system on the negative scattering length side, provided two-body bound states are absent, is enhanced the most compared to the energy of the system without spin-orbit coupling when $\tilde{\delta}=0$ or $q_{3, z}=-3 m \delta /\left(2 \hbar k_{\mathrm{so}}\right)$. The main text shows that this reasoning provides an intuitive understanding for the behavior of the lowest three-boson state in each manifold. However, the situation for the excited states in a manifold is more intricate [62].

\section{APPENDIX D: ATOM-DIMER THRESHOLD}

As already mentioned, the determination of the lowest atom-dimer scattering threshold requires knowledge of the dimer binding energy and the single-particle dispersion curve. Since the $z$ component of the center-of-mass momentum $q_{1, z}$ of the dimer, formed by particles 1 and 2, can be written as a linear combination of $q_{2, z}$ and $q_{3, z}, q_{1, z}$ is not a free parameter. As a consequence, the atom-dimer dispersion curves depend only on $q_{2, z}$ but not on $q_{1, z}$. Physically, this makes sense since the three-body system breaks up into two units (a dimer and an atom), with the momentum between the two units determining the division of the three-body momentum among the dimer and the atom.

To quantify this, we rewrite the Hamiltonian $\hat{\bar{H}}_{\text {rel }}$ by arbitrarily singling out the third atom and treating the expectation value $q_{2, z}$ of $\hat{q}_{2, z}$ as a parameter: 


$$
\begin{aligned}
\left.\hat{\bar{H}}_{\text {rel }}\right|_{\left\langle\hat{q}_{2, z}\right\rangle=q_{2, z}=} & \hat{H}_{12}\left(q_{2, z}\right) \otimes I_{3} \\
& +I_{1} \otimes I_{2} \otimes \hat{H}_{3}\left(q_{2, z}\right) \\
& +V_{\text {coupling. }}
\end{aligned}
$$

Here, the "dimer Hamiltonian" $\hat{H}_{12}\left(q_{2, z}\right)$ reads

$$
\begin{aligned}
\hat{H}_{12}\left(q_{2, z}\right)= & \left(\frac{\hat{\vec{q}}_{1}^{2}}{2 \mu_{1}}+V_{2 \mathrm{~b}}\left(r_{12}\right)\right) I_{1} \otimes I_{2} \\
& +\frac{\hbar k_{\mathrm{so}} q_{1, z}}{m}\left(\hat{\sigma}_{1, z} \otimes I_{2}-I_{1} \otimes \hat{\sigma}_{2, z}\right) \\
& +\left(\frac{\hbar k_{\mathrm{so}} q_{2, z}}{2 m}+\frac{\tilde{\delta}}{2}\right)\left(\hat{\sigma}_{1, z} \otimes I_{2}+I_{1} \otimes \hat{\sigma}_{2, z}\right) \\
& +\frac{\Omega}{2}\left(\hat{\sigma}_{1, x} \otimes I_{2}+I_{1} \otimes \hat{\sigma}_{2, x}\right) .
\end{aligned}
$$

Identifying $\tilde{\delta}_{12 \text {,eff }}$,

$$
\frac{\tilde{\delta}_{12, \mathrm{eff}}}{2}=\frac{\hbar k_{\mathrm{so}} q_{2, z}}{2 m}+\frac{\tilde{\delta}}{2},
$$

as a new effective dimer detuning, the eigenenergies of $\hat{H}_{12}\left(q_{2, z}\right)$ are the same as those of the two-body Hamiltonian. The "atom Hamiltonian" $\hat{H}_{3}\left(q_{2, z}\right)$,

$\hat{H}_{3}\left(q_{2, z}\right)=\frac{\vec{q}_{2}^{2}}{2 \mu_{2}} \otimes I_{3}+\left(-\frac{\hbar k_{\mathrm{so}} q_{2, z}}{m}+\frac{\tilde{\delta}}{2}\right) \hat{\sigma}_{3, z}+\frac{\Omega}{2} \hat{\sigma}_{3, x}$,

describes the Jacobi particle with mass $\mu_{2}$ and effective atom detuning $\tilde{\delta}_{3 \text {,eff }}$, where

$$
\frac{\tilde{\delta}_{3, \text { eff }}}{2}=-\frac{\hbar k_{\mathrm{so}} q_{2, z}}{m}+\frac{\tilde{\delta}}{2} .
$$

Note that the effective dimer detuning $\tilde{\delta}_{12, \text { eff }}$ and the effective atom detuning $\tilde{\delta}_{3 \text {,eff }}$ depend on the "true detuning" $\delta$, which is fixed by the experimental setup, on the $z$ component $q_{3, z}$ of the three-body center-of-mass momentum, which is a conserved quantity, and on $q_{2, z}$, which is treated as a parameter. Assuming that the distance between the center of mass of the dimer and the atom is large compared to the size of the dimer and compared to the ranges $r_{0}$ and $R_{0}$ of the two- and three-body interactions, the coupling term $V_{\text {coupling }}$,

$V_{\text {coupling }}=\left[V_{2 \mathrm{~b}}\left(r_{13}\right)+V_{2 \mathrm{~b}}\left(r_{23}\right)+V_{3 \mathrm{~b}}\left(r_{123}\right)\right] I_{1} \otimes I_{2} \otimes I_{3}$,

can be set to zero. Thus, the $q_{2, z}$-dependent relative atom-dimer dispersion curves are obtained by adding the

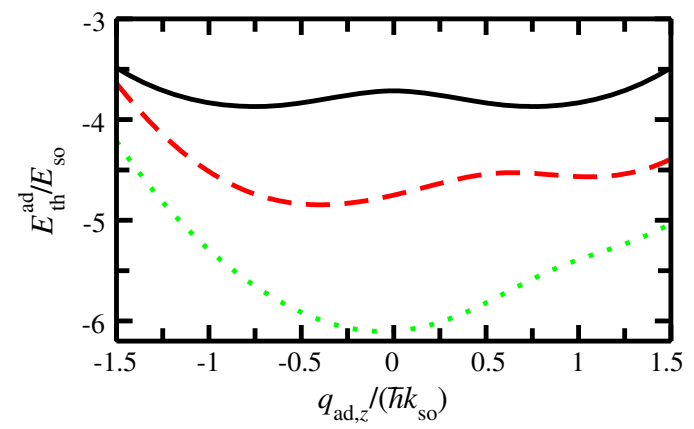

FIG. 6. Relative atom-dimer dispersion curves for $\left(a_{s} k_{\mathrm{so}}\right)^{-1}=$ 0.01128 and $\Omega / E_{\mathrm{so}}=2$. The solid, dashed, and dotted lines show the energy $E_{\text {th }}^{\text {ad }}$ as a function of the $z$ component $q_{\text {ad, } z}$ of the atomdimer momentum for $\tilde{\delta} / E_{\mathrm{so}}=0,2.287$, and 3.5, respectively.

eigenenergies of $\hat{H}_{12}$ and $\hat{H}_{3}$, which depend parametrically on $q_{2, z}$.

Equations (D1)-(D6) assume that the dimer is formed by atoms 1 and 2. Alternatively, the dimer could be formed by atoms 1 and 3 or by atoms 2 and 3. These alternative divisions yield atom-dimer dispersion curves that depend on the $z$ component of the momentum that is associated with the distance vector between particle 2 and the center of mass of the 13 dimer and the $z$ component of the momentum that is associated with the distance vector between particle 1 and the center of mass of the 23 dimer, respectively. Since we are considering three identical bosons, the three divisions are equivalent. In what follows, we use $q_{\text {ad,z }}$ to reflect that we could single out any of the three atoms. The corresponding atom-dimer energy is denoted by $E_{\text {th }}^{\text {ad }}$.

Since there exist up to three two-boson bound states [62], the three-boson system supports up to six atom-dimer dispersion curves (there could be four or two). As an example, Fig. 6 shows the energy $E_{\mathrm{th}}^{\mathrm{ad}}$ of the lowest relative atom-dimer dispersion curve as a function of $q_{\mathrm{ad}, z}$ for $\left(a_{s} k_{\mathrm{so}}\right)^{-1}=0.01128, \Omega / E_{\mathrm{so}}=2$, and various $\tilde{\delta}$, i.e., $\tilde{\delta}=0,2.287$, and 3.5. The system supports, for this Raman coupling strength and scattering length, one weakly bound two-boson state for all two-body center-of-mass momenta. For $\tilde{\delta}=0$ (solid line in Fig. 6), the atom-dimer dispersion is symmetric with respect to $q_{\mathrm{ad}, z}=0$ and supports two global minima at finite $q_{\mathrm{ad}, z}$. The breakup into a dimer and an atom is energetically most favorable when $q_{\text {ad, }, z} /\left(\hbar k_{\text {so }}\right)$ is equal to \pm 0.76 . This translates, using Eqs. (D3) and (D5), into $\tilde{\delta}_{12 \text {,eff }} / E_{\mathrm{so}}= \pm 1.52$ and $\tilde{\delta}_{3, \text { eff }} / E_{\mathrm{so}}= \pm 3.04$. For $\tilde{\delta}>0$ (the dashed and dotted lines in Fig. 6 are for $\tilde{\delta} / E_{\mathrm{so}}=2.287$ and 3.5, respectively), the atom-dimer dispersions are asymmetric with respect to $q_{\mathrm{ad}, z}=0$ and exhibit a global minimum at negative $q_{\mathrm{ad}, z}$, which approaches $q_{\text {ad, } z}=0$ in the $\tilde{\delta} \rightarrow \infty$ limit. Intuitively, the asymmetry can be understood by realizing that the atom and the dimer already see a detuning. Thus, moving in the 
positive momentum direction is not equivalent to moving in the negative momentum direction. The minimum of the lowest relative atom-dimer dispersion curve decreases with increasing $\tilde{\delta}$.

\section{APPENDIX E: THREE-BODY THRESHOLD}

The three-boson threshold is given by the minimum of the lowest three-atom threshold and the lowest atom-dimer threshold. It depends on the values of $\Omega, k_{\mathrm{so}}, \tilde{\delta}$, and the $s$-wave scattering length. Using $k_{\mathrm{so}}$ and $E_{\mathrm{so}}$ as units, Fig. 7 shows a contour plot of the lowest relative three-boson threshold as functions of the generalized detuning $\tilde{\delta} / E_{\mathrm{so}}$ and the inverse $\left(a_{s} k_{\mathrm{so}}\right)^{-1}$ of the $s$-wave scattering length for $\Omega / E_{\mathrm{so}}=2$. As already discussed, the parameter regime in which two-boson bound states exist depends on the value of $a_{s}$. Correspondingly, the thick dotted line, which marks the

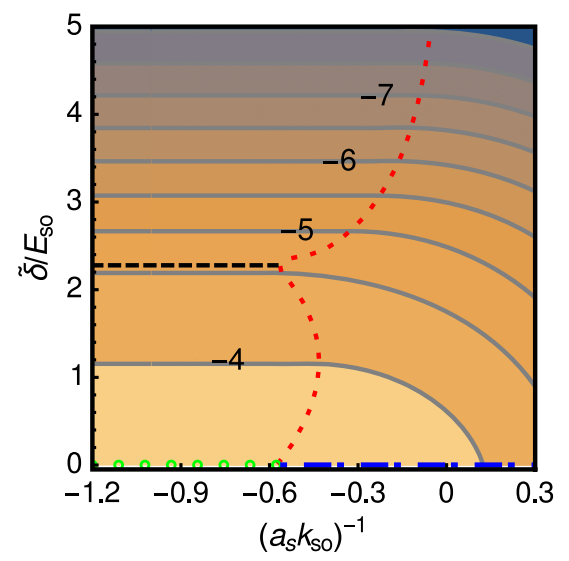

FIG. 7. Lowest relative three-boson scattering threshold for $\Omega / E_{\mathrm{so}}=2$. The contours show the energy $E_{\mathrm{th}}$, in units of $E_{\mathrm{so}}$, of the lowest three-boson scattering threshold as functions of $\left(a_{s} k_{\mathrm{so}}\right)^{-1}$ and $\tilde{\delta} / E_{\mathrm{so}}$. To the left of the thick dotted line, bound dimers are not supported and the lowest threshold is given by $E_{\mathrm{th}}^{\mathrm{aaa}}$. In this regime, the lowest scattering threshold is independent of $\left(a_{s} k_{\mathrm{so}}\right)^{-1}$. To the right of the thick dotted line, a weakly bound bosonic dimer exists and the lowest threshold is given by $E_{\mathrm{th}}^{\mathrm{ad}}$. In this regime, the lowest scattering threshold depends on $\left(a_{s} k_{\mathrm{so}}\right)^{-1}$. The thick open circles and thick dashed line mark the $\left[\left(a_{s} k_{\mathrm{so}}\right)^{-1}, \tilde{\delta} / E_{\mathrm{so}}\right]$ combinations for which the lowest three-atom threshold has a degeneracy of six and four, respectively. The thick dash-dotted line marks the $\left[\left(a_{s} k_{\mathrm{so}}\right)^{-1}, \tilde{\delta} / E_{\mathrm{so}}\right]$ combinations for which the lowest atom-dimer threshold has a degeneracy of two. In the region encircled by the thick open circles, the thick dotted line, the thick dashed line, and the left edge of the figure, the degeneracy of the three-atom threshold is equal to three. In the region encircled by the thick dashed line, the thick dotted line, the upper edge of the figure, and the left edge of the figure, the degeneracy of the three-atom threshold is equal to one. In the region encircled by the thick dash-dotted line, the right edge of the figure, the upper edge of the figure, and the thick dotted line, the degeneracy of the atom-dimer threshold is equal to one. The range of scattering lengths shown on the horizontal axis is, even though different units are used, the same as in Fig. 3. separation of the region in which the three-atom threshold has the lowest energy (to the left of the thick dotted line) and that in which the atom-dimer threshold has the lowest energy (to the right of the thick dotted line), shows a distinct dependence on the $s$-wave scattering length. For large $\tilde{\delta}$, the thick dotted line approaches the $\left(a_{s} k_{\mathrm{so}}\right)^{-1}=0$ line. For a fixed $\tilde{\delta}$, the energy $E_{\text {th }}^{\text {ad }}$ of the lowest atom-dimer threshold decreases with increasing $\left(a_{s} k_{\mathrm{so}}\right)^{-1}$. This can be traced back to the increase of the binding energy of the twoboson ground state with increasing $\left(a_{s} k_{\mathrm{so}}\right)^{-1}$. The parameter combinations with the largest degeneracy of the scattering threshold are shown by the thick open circles (three-atom threshold; the degeneracy is six) and the thick dash-dotted line (atom-dimer threshold; the degeneracy is two). For all $a_{s}$, the largest degeneracy of the scattering threshold is found for $\tilde{\delta}=0$. As we discuss in the main text, our numerical three-boson calculations show that the binding energy of the most strongly bound state in each manifold, determined as functions of $\left(a_{s} k_{\mathrm{so}}\right)^{-1}$ and $\tilde{\delta} / E_{\mathrm{so}}$, is largest for vanishing $\tilde{\delta}$, i.e., where the degeneracy of the lowest three-boson scattering threshold is maximal.

[1] H.-X. Chen, W. Chen, X. Liu, and S.-L. Zhu, The HiddenCharm Pentaquark and Tetraquark States, Phys. Rep. 639, 1 (2016).

[2] M. Gell-Mann, A Schematic Model of Baryons and Mesons, Phys. Lett. 8, 214 (1964).

[3] E. Epelbaum, H.-W. Hammer, and U.-G. Meißner, Modern Theory of Nuclear Forces, Rev. Mod. Phys. 81, 1773 (2009).

[4] I. Tanihata, Neutron Halo Nuclei, J. Phys. G 22, 157 (1996).

[5] L. H. Thomas, The Interaction between a Neutron and a Proton and the Structure of $\mathrm{H}^{3}$, Phys. Rev. 47, 903 (1935).

[6] V. Efimov, Energy Levels Arising from Resonant Two-Body Forces in a Three-Body System, Phys. Lett. B 33, 563 (1970).

[7] V. Efimov, Weakly-Bound States of Three ResonantlyInteracting Particles, Yad. Fiz. 12, 1080 (1970) [Sov. J. Nucl. Phys. 12, 589 (1971)].

[8] E. Braaten and H.-W. Hammer, Universality in Few-Body Systems with Large Scattering Length, Phys. Rep. 428, 259 (2006).

[9] E. Braaten and H.-W. Hammer, Efimov Physics in Cold Atoms, Ann. Phys. (Amsterdam) 322, 120 (2007).

[10] H.-W. Hammer and L. Platter, Efimov States in Nuclear and Particle Physics, Annu. Rev. Nucl. Part. Sci. 60, 207 (2010).

[11] P. Naidon and S. Endo, Efimov Physics: A Review, Rep. Prog. Phys. 80, 056001 (2017).

[12] F. Ferlaino and R. Grimm, Trend: Forty Years of Efimov Physics: How a Bizarre Prediction Turned into a Hot Topic, Physics 3, 9 (2010).

[13] C. H. Greene, Universal Insights from Few-Body Land, Phys. Today 63, No. 3, 40 (2010).

[14] T. Kraemer, M. Mark, P. Waldburger, J. G. Danzl, C. Chin, B. Engeser, A. D. Lange, K. Pilch, A. Jaakkola, H.-C. Nägerl, and R. Grimm, Evidence for Efimov Quantum States 
in an Ultracold Gas of Caesium Atoms, Nature (London) 440, 315 (2006).

[15] M. Zaccanti, B. Deissler, C. D’Errico, M. Fattori, M. JonaLasinio, S. Müller, G. Roati, M. Inguscio, and G. Modugno, Observation of an Efimov Spectrum in an Atomic System, Nat. Phys. 5, 586 (2009).

[16] Y. Nishida, Y. Kato, and C. D. Batista, Efimov Effect in Quantum Magnets, Nat. Phys. 9, 93 (2013).

[17] T. Lompe, T. B. Ottenstein, F. Serwane, A. N. Wenz, G. Zürn, and S. Jochim, Radio-Frequency Association of Efimov Trimers, Science 330, 940 (2010).

[18] S. Nakajima, M. Horikoshi, T. Mukaiyama, P. Naidon, and M. Ueda, Measurement of an Efimov Trimer Binding Energy in a Three-Component Mixture of ${ }^{6} \mathrm{Li}$, Phys. Rev. Lett. 106, 143201 (2011).

[19] M. Berninger, A. Zenesini, B. Huang, W. Harm, H.-C. Nägerl, F. Ferlaino, R. Grimm, P. S. Julienne, and J. M. Hutson, Universality of the Three-Body Parameter for Efimov States in Ultracold Cesium, Phys. Rev. Lett. 107, 120401 (2011).

[20] B. Huang, L. A. Sidorenkov, R. Grimm, and J. M. Hutson, Observation of the Second Triatomic Resonance in Efimov's Scenario, Phys. Rev. Lett. 112, 190401 (2014).

[21] M. Kunitski, S. Zeller, J. Voigtsberger, A. Kalinin, L. P. H. Schmidt, M. Schöffler, A. Czasch, W. Schöllkopf, R. E. Grisenti, T. Jahnke, D. Blume, and R. Dörner, Observation of the Efimov State of the Helium Trimer, Science 348, 551 (2015).

[22] D. C. Mattis, The Few-Body Problem on a Lattice, Rev. Mod. Phys. 58, 361 (1986).

[23] J. Dalibard, F. Gerbier, G. Juzeliūnas, and P. Öhberg, Colloquium: Artificial Gauge Potentials for Neutral Atoms, Rev. Mod. Phys. 83, 1523 (2011).

[24] V. Galitski and I. B. Spielman, Spin-Orbit Coupling in Quantum Gases, Nature (London) 494, 49 (2013).

[25] N. Goldman, G. Juzeliūnas, P. Öhberg, and I. B. Spielman, Light-Induced Gauge Fields for Ultracold Atoms, Rep. Prog. Phys. 77, 126401 (2014).

[26] H. Zhai, Degenerate Quantum Gases with Spin-Orbit Coupling: A Review, Rep. Prog. Phys. 78, 026001 (2015).

[27] M. Aidelsburger, S. Nascimbene, and N. Goldman, Artificial Gauge Fields in Materials and Engineered Systems, arXiv: 1710.00851.

[28] Y.-J. Lin, K. Jiménez-García, and I. B. Spielman, Spin-Orbit-Coupled Bose-Einstein Condensates, Nature (London) 471, 83 (2011).

[29] J. Struck, C. Ölschläger, M. Weinberg, P. Hauke, J. Simonet, A. Eckardt, M. Lewenstein, K. Sengstock, and P. Windpassinger, Tunable Gauge Potential for Neutral and Spinless Particles in Driven Optical Lattices, Phys. Rev. Lett. 108, 225304 (2012).

[30] C. V. Parker, L.-C. Ha, and C. Chin, Direct Observation of Effective Ferromagnetic Domains of Cold Atoms in a Shaken Optical Lattice, Nat. Phys. 9, 769 (2013).

[31] T. Ozawa, H. M. Price, A. Amo, N. Goldman, M. Hafezi, L. Lu, M. Rechtsman, D. Schuster, J. Simon, O. Zilberberg, and I. Carusotto, Topological Photonics, arXiv:1802.04173.

[32] J. P. Vyasanakere and V. B. Shenoy, Bound States of Two Spin-1/2 Fermions in a Synthetic Non-Abelian Gauge Field, Phys. Rev. B 83, 094515 (2011).
[33] J. P. Vyasanakere, S. Zhang, and V. B. Shenoy, BCS-BEC Crossover Induced by a Synthetic Non-Abelian Gauge Field, Phys. Rev. B 84, 014512 (2011).

[34] J. P. Vyasanakere and V. B. Shenoy, Rashbons: Properties and Their Significance, New J. Phys. 14, 043041 (2012).

[35] J. P. Vyasanakere and V. B. Shenoy, Flow-Enhanced Pairing and Other Unusual Effects in Fermi Gases in Synthetic Gauge Fields, Phys. Rev. A 88, 033609 (2013).

[36] X. Cui, Mixed-Partial-Wave Scattering with Spin-Orbit Coupling and Validity of Pseudopotentials, Phys. Rev. A 85, 022705 (2012).

[37] L. Dong, L. Jiang, H. Hui, and H. Pu, Finite-Momentum Dimer Bound State in a Spin-Orbit-Coupled Fermi Gas, Phys. Rev. A 87, 043616 (2013).

[38] Z. Yu, Short-Range Correlations in Dilute Atomic Fermi Gases with Spin-Orbit Coupling, Phys. Rev. A 85, 042711 (2012).

[39] Y. Wu and Z. Yu, Short-Range Asymptotic Behavior of the Wave Functions of Interacting Spin-1/2 Fermionic Atoms with Spin-Orbit Coupling: A Model Study, Phys. Rev. A 87, 032703 (2013).

[40] X. Cui, Multichannel Molecular State and Rectified ShortRange Boundary Condition for Spin-Orbit-Coupled Ultracold Fermions Near p-Wave Resonances, Phys. Rev. A 95, 030701(R) (2017).

[41] Q. Guan and D. Blume, Scattering Framework for Two Particles with Isotropic Spin-Orbit Coupling Applicable to All Energies, Phys. Rev. A 94, 022706 (2016).

[42] S.-J. Wang and C. H. Greene, General Formalism for Ultracold Scattering with Isotropic Spin-Orbit Coupling, Phys. Rev. A 91, 022706 (2015).

[43] D. Luo and L. Yin, BCS-Pairing State of a Dilute Bose Gas with Spin-Orbit Coupling, Phys. Rev. A 96, 013609 (2017).

[44] R. Li and L. Yin, Pair Condensation in a Dilute Bose Gas with Rashba Spin-Orbit Coupling, New J. Phys. 16, 053013 (2014).

[45] Z. Xu, Z. Yu, and S. Zhang, Evidence for Correlated States in a Cluster of Bosons with Rashba Spin-Orbit Coupling, New J. Phys. 18, 025002 (2016).

[46] Z.-Y. Shi, X. Cui, and H. Zhai, Universal Trimers Induced by Spin-Orbit Coupling in Ultracold Fermi Gases, Phys. Rev. Lett. 112, 013201 (2014).

[47] X. Cui and W. Yi, Universal Borromean Binding in Spin-Orbit-Coupled Ultracold Fermi Gases, Phys. Rev. X 4, 031026 (2014).

[48] Z.-Y. Shi, H. Zhai, and X. Cui, Efimov Physics and Universal Trimers in Spin-Orbit-Coupled Ultracold Atomic Mixtures, Phys. Rev. A 91, 023618 (2015).

[49] X. Qiu, X. Cui, and W. Yi, Universal Trimers Emerging from a Spin-Orbit-Coupled Fermi Sea, Phys. Rev. A 94, 051604(R) (2016).

[50] Q. Guan, One-, Two-, and Three-Body Systems with SpinOrbit Coupling, Ph.D. thesis, Washington State University, 2017.

[51] The normalization factor of the plane wave center-of-mass wave function is not written out for notational convenience.

[52] Y. Yan and D. Blume, Energy and Structural Properties of N-Boson Clusters Attached to Three-Body Efimov States: Two-Body Zero-Range Interactions and the Role of the Three-Body Regulator, Phys. Rev. A 92, 033626 (2015). 
[53] R. Pires, J. Ulmanis, S. Häfner, M. Repp, A. Arias, E. D. Kuhnle, and M. Weidemüller, Observation of Efimov Resonances in a Mixture with Extreme Mass Imbalance, Phys. Rev. Lett. 112, 250404 (2014).

[54] S.-K. Tung, K. Jiménez-García, J. Johansen, C. V. Parker, and C. Chin, Geometric Scaling of Efimov States in a ${ }^{6} \mathrm{Li}-{ }^{133}$ Cs Mixture, Phys. Rev. Lett. 113, 240402 (2014).

[55] F. Ferlaino, S. Knoop, M. Mark, M. Berninger, H. Schöbel, H.-C. Nägerl, and R. Grimm, Collisions between Tunable Halo Dimers: Exploring an Elementary Four-Body Process with Identical Bosons, Phys. Rev. Lett. 101, 023201 (2008).

[56] F. Ferlaino, S. Knoop, M. Berninger, W. Harm, J. P. D'Incao, H.-C. Nägerl, and R. Grimm, Evidence for Universal Four-Body States Tied to an Efimov Trimer, Phys. Rev. Lett. 102, 140401 (2009).

[57] S. E. Pollack, D. Dries, and R. G. Hulet, Universality in Three- and Four-Body Bound States of Ultracold Atoms, Science 326, 1683 (2009).

[58] A. Zenesini, B. Huang, M. Berninger, S. Besler, H.-C. Nägerl, F. Ferlaino, R. Grimm, C. H. Greene, and J. von Stecher, Resonant Five-Body Recombination in an Ultracold Gas of Bosonic Atoms, New J. Phys. 15, 043040 (2013).

[59] Spin-orbit-coupled experiments with Cs, which has tunable Feshbach resonances, have not yet been conducted. On the other hand, while many experiments utilize $\mathrm{Rb}$ to realize spin-orbit coupling, the known Feshbach resonances in $\mathrm{Rb}$ are accompanied by large losses, making the resonances challenging to use. Proposals for implementing spin-orbitcoupling schemes in Cs exist [60].

[60] B.-Z. Wang, Y.-H. Lu, W. Sun, S. Chen, Y. Deng, and X.-J. Liu, Dirac-, Rashba-, and Weyl-type Spin-Orbit Couplings: Toward Experimental Realization in Ultracold Atoms, Phys. Rev. A 97, 011605(R) (2018).

[61] When we apply our formalism to the two identical fermion system, our binding energy agrees with the results obtained by Vyasanakere and Shenoy [35] but disagrees with the results presented in Ref. [37]. Reference [37] calculated the two-fermion binding energy by allowing the $z$ component of the center-of-mass momentum of the eigenstate of the interacting system and that of the two-atom state associated with the threshold with respect to which the binding energy is being measured to be different. The definition employed [see Eq. (14) of Ref. [37]] implies that the $z$ component of the center-of-mass momentum is not a conserved quantity.
A straightforward calculation, however, establishes that the three components of the center-of-mass momentum are individually conserved (see, e.g., Ref. [50]). Correspondingly, the quantity considered in Ref. [37] is inconsistent with the accepted definition of the term "two-body binding energy." The quantity defined in Ref. [37] does, however, become meaningful in a many-body context. To see this, we consider a two-body bound state with total energy $E_{2}\left(\vec{q}_{2}\right)=$ $E+\vec{q}_{2}^{2} /(4 m)$ embedded into a many-body background (here, $E$ is the eigenenergy of the relative two-particle Hamiltonian; $E$ depends on $q_{2, z}$ ). Assuming that the dimer can reduce its energy via center of mass changing inelastic collisions with the background atoms, the lowest energy of the dimer is given by $\min _{\vec{q}_{2}} E_{2}\left(\vec{q}_{2}\right)$. The dimer can then be considered stable if the global energy $E_{\text {global,2, }}, E_{\text {global }, 2}=$ $\min _{\vec{q}_{2}} E_{2}\left(\vec{q}_{2}\right)-\min _{\vec{q}_{2}}\left[E_{\mathrm{th}}+\vec{q}_{2}^{2} /(4 m)\right]\left(E_{\mathrm{th}}\right.$ denotes the lowest relative atom-atom scattering threshold for fixed $q_{2, z}$ ) is negative. For the global energy $E_{\text {global,2 }}$, referred to as binding energy in Ref. [37], to be meaningful, the dimer needs to be embedded into a background that can, via inelastic collisions, absorb energy on a timescale that is fast compared to the timescales associated with other loss processes.

[62] Q. Guan and D. Blume (unpublished).

[63] N. T. Zinner, Efimov states of Heavy Impurities in a BoseEinstein Condensate, Europhys. Lett. 101, 60009 (2013).

[64] D. J. MacNeill and F. Zhou, Pauli Blocking Effect on Efimov States Near a Feshbach Resonance, Phys. Rev. Lett. 106, 145301 (2011).

[65] M. Gaulitis and R. Damburg, Some Features of the Threshold Behavior of the Cross Sections for Excitation of Hydrogen by Electrons due to the Existence of a Linear Stark Effect in Hydrogen, Sov. Phys. JETP 17, 1107 (1963).

[66] Y. Suzuki and K. Varga, Stochastic Variational Approach to Quantum-Mechanical Few-Body Problems, 1st ed. (Springer-Verlag, Berlin, 1998).

[67] J. Mitroy, S. Bubin, W. Horiuchi, Y. Suzuki, L. Adamowicz, W. Cencek, K. Szalewicz, J. Komasa, D. Blume, and K. Varga, Theory and Application of Explicitly Correlated Gaussians, Rev. Mod. Phys. 85, 693 (2013).

[68] D. Rakshit, K. M. Daily, and D. Blume, Natural and Unnatural Parity States of Small Trapped Equal-Mass Two-Component Fermi Gases at Unitarity and FourthOrder Virial Coefficient, Phys. Rev. A 85, 033634 (2012). 